ZMP-HH/13-13

Hamburger Beiträge zur Mathematik Nr. 484

July 2013

\title{
A geometric approach to boundaries and surface defects in Dijkgraaf-Witten theories
}

\author{
Jürgen Fuchs ${ }^{a}$, Christoph Schweigert $^{b}$, Alessandro Valentino ${ }^{b}$ \\ ${ }^{a}$ Teoretisk fysik, Karlstads Universitet \\ Universitetsgatan 21, S-65188 Karlstad \\ ${ }^{b}$ Fachbereich Mathematik, Universität Hamburg \\ Bereich Algebra und Zahlentheorie \\ Bundesstraße 55, D-20146 Hamburg
}

\begin{abstract}
Dijkgraaf-Witten theories are extended three-dimensional topological field theories of TuraevViro type. They can be constructed geometrically from categories of bundles via linearization. Boundaries and surface defects or interfaces in quantum field theories are of interest in various applications and provide structural insight. We perform a geometric study of boundary conditions and surface defects in Dijkgraaf-Witten theories. A crucial tool is the linearization of categories of relative bundles. We present the categories of generalized Wilson lines produced by such a linearization procedure. We establish that they agree with the Wilson line categories that are predicted by the general formalism for boundary conditions and surface defects in three-dimensional topological field theories that has been developed in [FSV].
\end{abstract}




\section{Introduction}

For more than two decades, Dijkgraaf-Witten theories have provided a laboratory for new ideas in mathematical physics. They form a particularly tractable subclass of three-dimensional topological field theories. Since they have a Lagrangian description in which path integrals reduce to counting measures, they also serve as toy models for more complicated classes of topological field theories like Chern-Simons theories.

The defining data of a Dijkgraaf-Witten theory are a finite group $G$ and a 3-cocycle $\omega \in Z^{3}\left(G, \mathbb{C}^{\times}\right)$. Given these data, a clear geometric construction [Fr, Mor] describes the theory in terms of a linearization of categories of spans of $G$-bundles. In the present paper we extend this approach by a geometric study of Dijkgraaf-Witten theories on manifolds with boundaries and defects. More specifically, we consider the class of boundary conditions and defects for three-dimensional topological field theories that was investigated in [FSV]. Besides providing new structural insight, such boundary conditions and surface defects are relevant to various applications, ranging from a geometric visualization of the TFT approach to RCFT correlators to universality classes of gapped boundaries and defects in condensed matter systems that are of interest in many areas.

A crucial input in our construction are the concepts of relative manifolds and relative bundles. Via the linearization of relative bundles we obtain categories of generalized Wilson lines for Dijkgraaf-Witten theories with boundaries and defects. Our results perfectly match the general analysis of [FSV], combined with Ostrik's explicit description Os2 of module categories over the categories of $G$-graded vector spaces.

The rest of this paper is organized as follows. In Section 2 we collect pertinent background information. We start in Section 2.1 with a summary of the geometric construction of DijkgraafWitten theories, with emphasis on the implementation of locality, which naturally leads to the use of bicategories. We then present some facts about relative bundles (Section 2.3), about groupoid cohomology (Section 2.4), and about module categories over the monoidal category $G$-vect ${ }^{\omega}$ of $G$-graded vector spaces with associativity constraint twisted by the cocycle $\omega$ (Section 2.5).

Section 3 contains our results for categories of generalized Wilson lines in Dijkgraaf-Witten theories with defects and boundaries. These categories are associated to one-dimensional manifolds with additional data. In the present paper, we restrict our attention to one-dimensional manifolds, leaving the case of two-dimensional manifolds with boundaries and of three-dimensional manifolds with corners to future work. (The results for two- and three-dimensional manifolds will allow us to make statements about generalized partition functions.) In Section 3.1 we discuss the relevant concepts of decorated one-dimensional manifolds and of categories of generalized bundles and use them to obtain the groupoids for the geometric situations of our interest. Afterwards we introduce in Section 3.2 the additional data from groupoid cohomology that are needed for the linearization process. From the perspective of Lagrangian field theory, these data are a topological bulk Lagrangian and compatible boundary terms; accordingly we refer to them as Lagrangian data. In section 3.3 we explain how to get 2-cocycles for the groupoids obtained in Section 3.1 from Lagrangian data assigned to intervals and circles.

Invoking fusion of defects, all one-dimensional manifolds arising from boundaries and defects can be reduced to two building blocks: the interval without interior marked points, and the circle with a single marked point. The linearization of the groupoids for these two basic situations is described in detail in Section 3.5 and 3.7, respectively. A convenient tool in these calculations is 
a graphical calculus for groupoid cocycles which is inspired by [Wi]. It is introduced in Section 3.4. Another input is a concrete description of the transparent surface defect; this is obtained in Section 3.6, based crucially on the invariance of the graphical calculus under Pachner moves.

In the considerations in Sections 3.5 and 3.7 we concentrate on the situation that the relevant group homomorphisms are subgroup embeddings; these lead to indecomposable module categories over $G$-vect ${ }^{\omega}$. Without this restriction, one obtains decomposable module categories; this is discussed in the Appendix.

\section{Background material}

In this section we summarize some background material on the geometric construction of Dijkgraaf-Witten theories and on boundaries and surface defects in three-dimensional topological field theories, and on some aspects of relative bundles.

We fix the following conventions. By vect $_{\mathbb{k}}$ we denote the category of finite-dimensional vector spaces over a field $\mathbb{k}$; In the present paper we only consider the case of complex vector spaces, $\mathbb{k}=\mathbb{C}$. A group is assumed to be finite. Manifolds, including manifolds with boundaries and manifolds with corners, are smooth.

For a finite group $G$ and a smooth manifold $X$ of any dimension, we denote by $\operatorname{Bun}_{G}(X)$ the category of smooth $G$-principal bundles, which has maps covering the identity as morphisms. We adopt the convention that the $G$-action on the fiber of a principal $G$-bundle is a right action. In particular, a $G$-bundle over a point is just a right $G$-torsor. Morphisms of the category $\operatorname{Bun}_{G}(X)$ are morphisms of $G$-bundles covering the identity. They are all invertible, i.e. $\operatorname{Bun}_{G}(X)$ is a groupoid. Diffeomorphisms $f: X \rightarrow Y$ relate the groupoids by pullback functors, $f^{*}: \operatorname{Bun}_{G}(Y) \rightarrow \operatorname{Bun}_{G}(X)$. We note that with respect to e.g. surjective submersions, $\operatorname{Bun}_{G}$ becomes a stack on the category of smooth manifolds; we will not use the language of stacks in this paper, though.

\subsection{The geometric construction of Dijkgraaf-Witten theories}

A classic definition by Atiyah characterizes $d$-dimensional topological field theories as symmetric monoidal functors from a geometric category, the symmetric monoidal category cobord ${ }_{d, d-1}$ of $d$-dimensional cobordisms, to some linear category, e.g. to the symmetric monoidal category vect $_{\mathbb{C}}$. A classic result states that for $d=2$ the functor given by

$$
\mathrm{tft} \longmapsto \operatorname{tft}\left(S^{1}\right)
$$

is an equivalence between the category of topological field theories and the category of complex commutative Frobenius algebras.

Dijkgraaf-Witten theories are three-dimensional topological field theories. The DijkgraafWitten theory

$$
\operatorname{tft}_{G}: \operatorname{cobord}_{3,2} \rightarrow \operatorname{vect}_{\mathbb{C}}
$$

based on a finite group $G$ can be characterized as follows. The functor $\mathrm{tft}_{G}$ associates to a closed oriented surface $\Sigma$ the vector space $\operatorname{tft}_{G}(\Sigma)$ freely generated by the set of isomorphism 
classes of principal G-bundles on $\Sigma$. To a cobordism

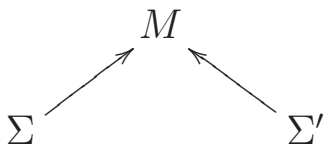

it associates a linear map $\operatorname{tft}_{G}(\Sigma) \rightarrow \operatorname{tft}_{G}\left(\Sigma^{\prime}\right)$ whose matrix element for principal $G$-bundles $P$ on $\Sigma$ and $P^{\prime}$ on $\Sigma^{\prime}$ is the number $\left|\operatorname{Bun}_{G}\left(M, P, P^{\prime}\right)\right|$. Here $\operatorname{Bun}_{G}\left(M, P, P^{\prime}\right)$ is the groupoid of $G$-bundles on $M$ that restrict to a given $G$-bundle $P$ on $\Sigma$ and to $P^{\prime}$ on $\Sigma^{\prime}$, and for any groupoid $\Gamma$ we denote by $|\Gamma|$ the groupoid cardinality, which is the rational number

$$
|\Gamma|:=\sum_{\gamma \in \pi_{0}(\Gamma)} \frac{1}{|\operatorname{Aut}(\gamma)|}
$$

obtained by summing over the set $\pi_{0}(\Gamma)$ of isomorphism classes of objects of $\Gamma$.

The introduction of $d$-1-dimensional manifolds can be seen as a first step towards implementing locality in topological field theories: These submanifolds can be used to cut the $d$-dimensional manifold into smaller and simpler pieces, which are manifolds with boundary. The boundaries of cobordisms are thus to be thought of as "cut-and-paste boundaries". They must not be mixed up with physical boundaries to be discussed in section 2.2 .

Our analysis uses a framework which goes one step further in the implementation of locality and naturally leads to the use of bicategories. We need the following concepts:

\section{Definition 2.1.}

(i) The bicategory 2 -vect $_{\mathbb{C}}$ of complex 2 -vector spaces is the bicategory of $\mathbb{C}$-linear finitely semisimple abelian categories.

The Deligne product of $\mathbb{C}$-linear categories endows this bicategory with the structure of a symmetric monoidal bicategory.

(ii) The symmetric monoidal category $\operatorname{cobord}_{3,2,1}$ has as objects compact oriented smooth one-dimensional manifolds. 1-morphisms are two-dimensional manifolds with boundary; 2morphisms are three-manifolds with corners, up to diffeomorphisms preserving the orientation and the boundary. (For brevity we suppress collars in our discussion.)

(iii) An extended three-dimensional topological field theory is a symmetric monoidal functor

$$
\text { tft : } \operatorname{cobord}_{3,2,1} \rightarrow 2 \text {-vect }_{\mathbb{C}} .
$$

We note that, as a consequence of the axioms,

$$
\operatorname{tft}\left(S \sqcup S^{\prime}\right) \cong \operatorname{tft}(S) \otimes \operatorname{tft}\left(S^{\prime}\right)
$$

for any pair $\left(S, S^{\prime}\right)$ of one-dimensional manifolds, and $\operatorname{tft}(\emptyset)=\operatorname{vect}_{\mathbb{C}}$, where $\emptyset$ is considered as a one-dimensional manifold and monoidal unit of $\operatorname{cobord}_{3,2,1}$.

The Dijkgraaf-Witten theory based on a finite group $G$ is in fact an extended topological field theory [Fr, Mor]. It assigns to a one-dimensional manifold $S$ the category

$$
\operatorname{tft}_{G}(S):=\left[\operatorname{Bun}_{G}(S), \operatorname{vect}_{\mathbb{C}}\right]
$$

Here by $\left[\mathcal{C}_{1}, \mathcal{C}_{2}\right]$ we denote the category of functors between two (essentially small) categories $\mathcal{C}_{1}$ and $\mathcal{C}_{2}$. 
This formula already gives a hint on the general construction of the theory: In a first step, one uses the functor $\mathrm{Bun}_{G}$ that associates to a smooth manifold the groupoid of $G$-bundles to construct a bifunctor

$$
\operatorname{cobord}_{3,2,1} \stackrel{\widetilde{\operatorname{Bun}}_{G}}{\longrightarrow} \text { SpanGrp }
$$

to a bicategory of spans of groupoids. In a second step one linearizes by taking functor categories with values in vect $_{\mathbb{C}}$,

$$
\mathrm{tft}_{G}: \quad \operatorname{cobord}_{3,2,1} \stackrel{\widetilde{\operatorname{Bun}}_{G}}{\longrightarrow} \operatorname{SpanGrp} \stackrel{\left[-, \text { vect }_{C}\right]}{\longrightarrow} 2 \text {-vect } \text { v } .
$$

The non-extended topological field theory can be obtained from this extended topological field theory by restricting to the endomorphism categories of the monoidal units of $\operatorname{cobord}_{3,2,1}$ and 2 -vect ${ }_{\mathbb{C}}$, since $\operatorname{End}_{\text {cobord } 3,2,1}(\emptyset) \cong \operatorname{cobord}_{3,2}$ and $\operatorname{End}_{2 \text {-vect }}\left(\right.$ vect $\left._{\mathbb{C}}\right) \cong \operatorname{vect}_{\mathbb{C}}$.

The fact that $\mathrm{tft}_{G}$ involves pure counting measures amounts to considering vanishing Lagrangians. Dijkgraaf and Witten [DW] introduced the following generalization, in which the linearization is only projective. Select a cocycle $\omega$ representing a class $[\omega] \in H^{3}\left(G, \mathbb{C}^{\times}\right)$in group cohomology. One may think about this class as a 2-gerbe Wi on the classifying space $B G$ of $G$-bundles, which we represent by the action groupoid $* / / G$ of $G$ acting on a single object *. A $G$-bundle on a 3-manifold $M$ corresponds to a map into this classifying space. Pulling back the 2-gerbe along this map to $M$ we get a 2-gerbe on $M$, which for dimensional reasons is trivial. It therefore gives rise to a 3-manifold holonomy, which should be seen as the value of a topological Lagrangian. For this reason, we refer to the cocycle $\omega$ (and later on to similar quantities) as a Lagrangian datum.

The second step of the construction of Dijkgraaf-Witten models consists of a linearization of the groupoids obtained in the first step. In general, such a linearization is only projective. The relevant 2-cocycle on the groupoids must be derived from the Lagrangian data. In the case at hand, the 3-cocycle $\omega$ can be transgressed [Wi] to a cocycle $\tau(\omega)$ representing a class in $H^{2}\left(G / / G, \mathbb{C}^{\times}\right)$, the groupoid cohomology for the action groupoid $G / /_{\text {ad }} G$ with $G$ acting on itself by the adjoint action.

Direct calculation now yields $\mathrm{Mor} \operatorname{tft}_{G}\left(S^{1}\right)=\mathcal{D}^{\omega}(G)$-mod, i.e. the category associated to the circle is the modular tensor category of modules over the twisted Drinfeld double [DPR] of the category of $G$-graded vector spaces - or, equivalently, of complex representations of the finite group $G$. This category is the category of bulk Wilson lines. The goal of the present paper is to generalize this construction to more general cobordism categories and to consistently obtain categories of generalized Wilson lines: both bulk and boundary Wilson lines. Our construction requires the use of more general categories of bundles on smooth manifolds.

\subsection{Boundaries and defects in three-dimensional TFT}

The structure of boundary conditions in two-dimensional topological field theories is well understood [LaP, $\mathrm{MoS}]$ in the framework of open/closed topological field theories. In this setting one considers a larger cobordism category cobord ${ }_{2,1}^{\mathrm{op} / \mathrm{cl}}$. Its objects are one-dimensional smooth manifolds with boundary, with a suitable boundary condition fixed for each connected component of the (physical) boundary. Morphisms are now cobordisms with boundary, with each boundary component partitioned into segments each of which is either a physical boundary or a cut-and-paste boundary. An open/closed topological field theory is then a symmetric monoidal 
functor cobord ${ }_{2,1}^{\mathrm{op} / \mathrm{cl}} \rightarrow$ vect $_{\mathbb{C}}$. It turns out that a boundary condition $a$ gives rise to a (not necessarily commutative) Frobenius algebra $W_{a}$ whose center is the commutative Frobenius algebra $\operatorname{tft}\left(S^{1}\right)$. Explicitly, a boundary condition is thus a pair consisting of a Frobenius algebra $W_{a}$ and an isomorphism

$$
\operatorname{tft}\left(S^{1}\right) \stackrel{\cong}{\longrightarrow} Z\left(W_{a}\right)
$$

of commutative associative algebras. Once such a Frobenius algebra $W_{a}$ has been determined, the category of boundary conditions can be described as the category $W_{a}$-mod.

We pause for two comments. First, we allow for point-like insertions on boundaries that separate possibly different boundary conditions. As a consequence, boundary conditions form a category rather than a set: The space $\operatorname{Hom}_{W_{a}-\bmod }\left(M_{c}, M_{d}\right)$ of morphisms between two boundary conditions $M_{c}, M_{d} \in W_{a}$-mod is the vector space of labels for insertions that separate the boundary condition $M_{c}$ from the boundary condition $M_{d}$. Second, distinguishing one boundary condition in the discussion could be avoided, but at the price of using a higher-categorical language: the one of module categories over vect $\mathbb{C}$. For the three-dimensional topological field theories we are interested in, a Morita invariant treatment would amount to working with three-categories; we prefer an approach that avoids this. For a more detailed analysis of two-dimensional open/closed topological field theories we refer to the literature, in particular to $[\mathrm{LaP}]$.

Once one allows for manifolds with boundary, codimension-one defects that partition a manifold into cells supporting possibly different topological field theories are a natural extension of the picture described above. For two-dimensional theories such defects provide a lot of additional insight, in particular about symmetries and dualities [FFRS].

In three-dimensional topological field theories, boundary conditions and defects have been studied only recently. In this case, codimension-one defects are surface defects. Boundaries and surface defects in three-dimensional topological field theories of Reshetikhin-Turaev type appear in a geometric interpretation [KaS] of the TFT approach [SFR] to RCFT correlators and as models for universality classes of gapped boundaries and gapped interfaces for topological phases (see e.g. KK,WW,Le BJQ, Ka ), which arise for instance in the study of 2+1-dimensional electron fluids, including certain fractional quantum Hall states.

A model-independent study of boundary conditions and surface defects in such theories [FSV] yields the following results, which can be regarded as a categorified version of the results in two dimensions described above. To any boundary condition $a$ there is associated a fusion category $\mathcal{W}_{a}$. It describes boundary Wilson lines, i.e. Wilson lines that are confined to the boundary with boundary condition $a$. Let us recall that, depending on the chosen formalism, Wilson lines are embedded ribbons or tubes with a marked line at the boundary of the tube. In a similar spirit, boundary Wilson lines should be described by half-tubes extending into the 
three-dimensional bulk, as illustrated by the following picture:
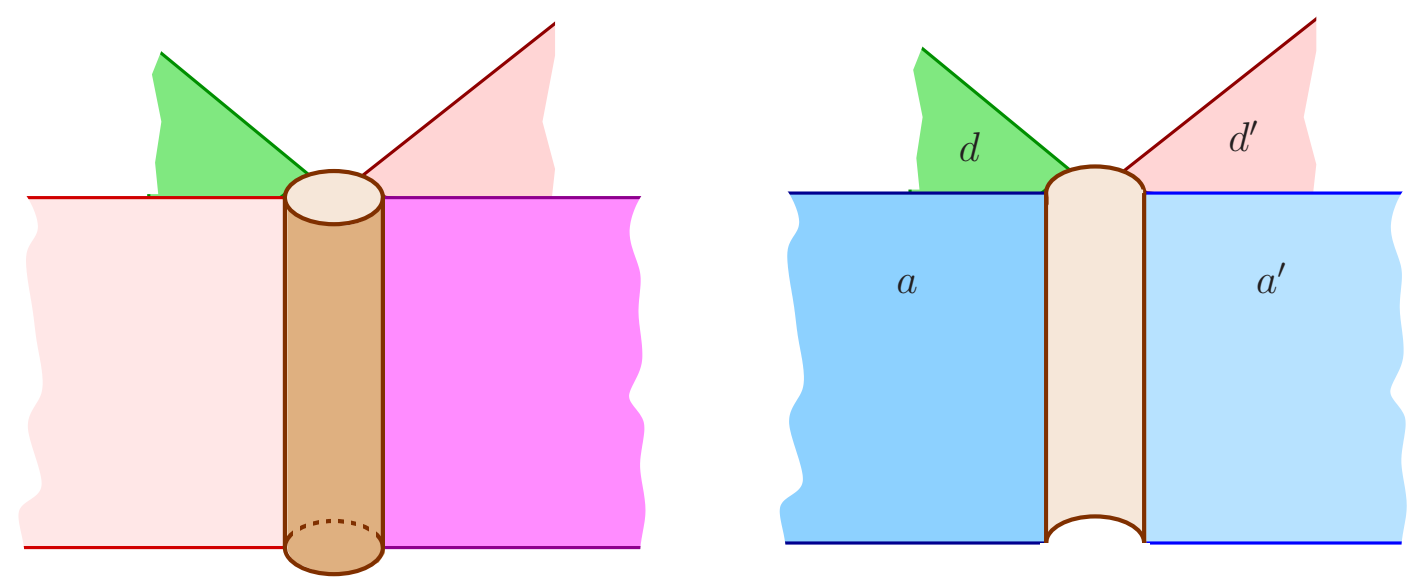

Here the figure on the right shows a boundary Wilson line in the form of a half-tube separating two (possibly different) boundary conditions $a$ and $a^{\prime}$ and at which two surface defects $d$ and $d^{\prime}$ end, while the left figure shows a bulk Wilson line in the form of a tube at which four surface defects end.

Since boundary Wilson lines are objects in a two-dimensional theory, the category $\mathcal{W}_{a}$ is not braided. A boundary condition can now be defined as a pair consisting of a fusion category $\mathcal{W}_{a}$ and a braided equivalence

$$
\mathcal{C}=\operatorname{tft}\left(S^{1}\right) \stackrel{\simeq}{\longrightarrow} \mathcal{Z}\left(\mathcal{W}_{a}\right),
$$

where $\mathcal{Z}$ denotes the Drinfeld center of the fusion category $\mathcal{W}_{a}$, which is a braided monoidal category. We refer to an equivalence of the type (2.12) as a Witt trivialization of $\mathcal{C}$. One should note that not any braided category is equivalent to a Drinfeld center. In general three-dimensional topological field theories this is a source of obstructions. But in the case of Dijkgraaf-Witten theories the relevant modular tensor category $\mathcal{C}$ indeed is a Drinfeld double, namely the Drinfeld double of the fusion category $G$-vect ${ }^{\omega}$ of $G$-graded vector spaces with associator twisted by $\omega$ (see Section 2.5)

$$
\mathcal{C}=\mathcal{Z}\left(G \text {-vect }{ }^{\omega}\right) .
$$

As a consequence, in the case of our interest the existence of boundary conditions is not obstructed.

The collection of all boundary conditions now has the structure of a bicategory: the bicategory of all module categories over the fusion category $\mathcal{W}_{a}$. (Module categories over a fusion category are a categorification of the notion of a module over a ring; we refer to Os1 for details.) The category of boundary Wilson lines separating two boundary conditions $c$ and $d$ that are given by two $\mathcal{W}_{a}$-module categories $\mathcal{M}_{c}$ and $\mathcal{M}_{d}$, respectively, is the abelian $\mathbb{C}$-linear category

$$
\operatorname{Fun}_{\mathcal{W}_{a}-\bmod }\left(\mathcal{M}_{c}, \mathcal{M}_{d}\right)
$$

of $\mathcal{W}_{a}$-module functors.

A similar analysis can be carried out for surface defects that separate two topological field theories of Reshetikhin-Turaev type, which are labeled by modular tensor categories $\mathcal{C}_{1}$ and $\mathcal{C}_{2}$. The category of Wilson lines in a surface defect of type $d$ is now a fusion category $\mathcal{W}_{d}$ together with a braided equivalence

$$
\mathcal{C}_{1} \otimes \mathcal{C}_{2}^{\text {rev }} \stackrel{\simeq}{\longrightarrow} \mathcal{Z}\left(\mathcal{W}_{d}\right) .
$$


Since the modular categories relevant for Dijkgraaf-Witten theories are already Drinfeld centers themselves, the existence of surface defects between any two Dijkgraaf-Witten theories is not obstructed. The category of Wilson lines separating surface defects that are given by two $\mathcal{W}_{d}$-module categories $\mathcal{M}_{c}$ and $\mathcal{M}_{d}$, respectively, is the abelian $\mathbb{C}$-linear category

$$
\operatorname{Fun}_{\mathcal{W}_{a}-\bmod }\left(\mathcal{M}_{c}, \mathcal{M}_{d}\right)
$$

of $\mathcal{W}_{d}$-module functors.

In the special case of defects separating a modular tensor category $\mathcal{C}$ from itself, we can work with the canonical Witt trivialization

$$
\text { can : } \quad \mathcal{C} \otimes \mathcal{C}^{\text {rev }} \stackrel{\simeq}{\longrightarrow} \mathcal{Z}(\mathcal{C}) .
$$

This functor maps the object $U \otimes V \in \mathcal{C} \otimes \mathcal{C}^{\text {rev }}$ to the object $U \otimes V \in \mathcal{C}$ endowed with a half braiding $e_{U \otimes V}$ given by [ENO, Eq. (4.2)]

$$
e_{U \otimes V}(X): \quad U \otimes V \otimes X \stackrel{c^{-1}}{\longrightarrow} U \otimes X \otimes V \stackrel{c}{\longrightarrow} X \otimes U \otimes V .
$$

With respect to the canonical Witt trivialization (2.17), we describe a defect separating $\mathcal{C}$ from itself by a $\mathcal{C}$-module category. Now $\mathcal{C}$ has a natural structure of module category over itself. This specific $\mathcal{C}$-module category describes a particularly important surface defect, the transparent (or invisible) surface defect. In fact, one expects a notion of a fusion product of defects, so that the bicategory of surface defects is even a monoidal bicategory. The transparent defect is then the tensor unit of the monoidal bicategory of defects. (At one step lower in the categorical ladder, the tensor unit of the monoidal category of endofunctors of any given defect category describes a Wilson line that is invisible inside the surface. The category of endofunctors of $\mathcal{C}$ describes Wilson lines inside the transparent defect; these are ordinary bulk Wilson lines. In particular, the tensor unit of this monoidal category is the invisible bulk Wilson line.)

Our goal in this paper is to achieve a concrete geometric, Lagrangian construction of some of the categories describing Wilson lines in the presence of boundaries and surface defects in Dijkgraaf-Witten theories in the spirit of [Fr, Mor]. To this end, we need the appropriate geometric objects that form categories whose linearizations enter in the topological field theory.

\subsection{Relative bundles}

In this section we review the notion of a relative bundle. We restrict our attention to finite groups, which is sufficient for our construction.

\section{Definition 2.2.}

Let $G$ and $H$ be finite groups, $\iota: H \rightarrow G$ a morphism of finite groups, and $X$ a smooth manifold, Then the functor

$$
\operatorname{Ind}_{\iota}: \operatorname{Bun}_{H}(X) \rightarrow \operatorname{Bun}_{G}(X)
$$

is the one that acts on objects as $P_{H} \mapsto P_{H} \times_{H} G$.

\section{Remark 2.3.}

(i) If the group homomorphism $\iota$ injective, then the functor $\operatorname{Ind}_{\iota}$ is injective on morphisms. 
Indeed, suppose $f_{1}, f_{2}: P_{H} \rightarrow P_{H}^{\prime}$ are two different morphisms of $H$-bundles on $X$. Then there exist points $x \in X$ and $p$ in the fiber of $P_{H}$ over $x$ such that $f_{1}(p) \neq f_{2}(p)$. Since both $f_{1}(p)$ and $f_{2}(p)$ are in the fiber of $P_{H}^{\prime}$ over $x$, we have a unique $h \in H \backslash\{e\}$ such that $f_{1}(p)=f_{2}(p)$. $h$. Suppose that after induction $\left[f_{1}(p), g\right]=\left[f_{2}(p), g\right]$ for some $g \in G$. Then

$$
\left[f_{1}(p), g\right]=\left[f_{2}(p), g\right]=\left[f_{1}(p) \cdot h, g\right]=\left[f_{1}(p), \iota(h) \cdot g\right]
$$

Equality of the left and right hand sides implies $\iota(h) \cdot g=g$, i.e. $\iota(h)=e$. If $\iota$ is injective, this is impossible for $h \neq e$.

(ii) Induction commutes with pullback: if $f: X_{1} \rightarrow X_{2}$ is a morphism of smooth manifolds and if $P_{H}^{(2)}$ is a $H$-bundle on $X_{2}$, then

$$
\operatorname{Ind}_{\iota} f^{*} P_{H}^{(2)}=f^{*} \operatorname{Ind}_{\iota} P_{H}^{(2)}
$$

More abstractly, for any finite group $G$ we have the stack $\operatorname{Bun}_{G}(-)$ of $G$-bundles on the category of smooth manifolds with topology given by surjective submersions. Induction is also compatible with descent. Thus $\operatorname{Ind}_{\iota}$ gives a morphism $\operatorname{Ind}_{\iota}: \operatorname{Bun}_{H} \rightarrow \operatorname{Bun}_{G}$ of stacks.

A crucial ingredient for our construction is the notion of relative smooth manifolds and relative bundles. This is as follows, see e.g. St].

\section{Definition 2.4.}

(i) A relative (smooth) manifold $Y \stackrel{j}{\rightarrow} X$ consists of a pair $Y, X$ of smooth manifolds and a morphism $j: Y \rightarrow X$ of smooth manifolds.

A morphism $\left(Y_{1} \stackrel{j_{1}}{\rightarrow} X_{1}\right) \longrightarrow\left(Y_{2} \stackrel{j_{2}}{\rightarrow} X_{2}\right)$ of relative smooth manifolds is a commuting diagram

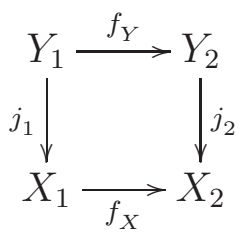

in the category of smooth manifolds.

(ii) Let $\iota: H \rightarrow G$ be a homomorphism of finite groups. A relative $(G, H)$-bundle on the relative manifold $Y \stackrel{j}{\rightarrow} X$ is a triple consisting of a $G$-bundle $P_{G}$ on $X$, an $H$-bundle $P_{H}$ on $Y$, and an isomorphism

$$
\alpha: \quad \operatorname{Ind}_{\iota}\left(P_{H}\right) \stackrel{\simeq}{\longrightarrow} j^{*}\left(P_{G}\right)
$$

of $G$-bundles on $Y$.

(iii) A morphism $\left(P_{G}, P_{H}, \alpha\right) \rightarrow\left(P_{G}^{\prime}, P_{H}^{\prime}, \alpha^{\prime}\right)$ of relative $(G, H)$-bundles on a relative smooth manifold $Y \stackrel{j}{\rightarrow} X$ consists of a morphism

$$
\varphi_{G}: \quad P_{G} \rightarrow P_{G}^{\prime}
$$

of $G$-bundles on $X$ and of a morphism

$$
\varphi_{H}: \quad P_{H} \rightarrow P_{H}^{\prime}
$$


of $H$-bundles on $Y$ such that the diagram

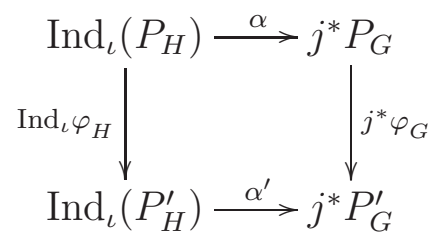

of morphisms of $G$-bundles on $Y$ commutes.

The category of relative $(G, H)$-bundles on $(X, Y)$ is denoted by $\operatorname{Bun}_{(G, H)}(Y \rightarrow X)$.

\section{Remark 2.5.}

(i) The category $\operatorname{Bun}_{(G, H)}(Y \rightarrow X)$ depends the group homomorphism $\iota: H \rightarrow G$. The notation $\operatorname{Bun}_{(G, H)}(Y \rightarrow X)$ suppresses this dependence and is thus slightly inappropriate.

(ii) The category $\operatorname{Bun}_{(G, H)}(Y \rightarrow X)$ inherits from the category of principal bundles the property of being a groupoid: all morphisms of relative bundles are invertible.

(iii) For the special case that $j=i d_{X}$ is the identity on $X=Y$, we obtain the notion of a reduction of a $G$-bundle to an $H$-bundle along the group homomorphism $\iota$.

(iv) As an object, a relative bundle is thus a $G$-bundle $P_{G}$ on $X$ together with a reduction of its pullback $j^{*} P_{G}$ to an $H$-bundle along the group homomorphism $\iota$. One should note, however, that the morphisms in $\operatorname{Bun}_{G, H}(X, Y)$ are not simply morphisms of reductions, which would only involve a morphism of $G$-bundles on the manifold $Y$. Rather, also a $G$-morphism on the manifold $X$ is required. (Later on, $Y$ will typically be a submanifold of $X$; hence we require a morphism on a larger manifold in that case.) In gauge theory terminology, the morphisms are thus gauge transformations on $Y$ and on $X$, respectively.

(v) If the group homomorphism $\iota$ is injective, then by remark 2.3(i) the morphism $\varphi_{H}$ of $H$-bundles is determined uniquely by $\varphi_{G}$, provided it exists. It is thus not an extra datum. The morphisms of relative $(G, H)$-bundles are in this situation morphisms of $G$-bundles that are compatible with the reductions.

(vi) Fix a homomorphism $\iota: H \rightarrow G$ of finite groups and consider a relative bundle $\left(P_{G}^{2}, P_{H}^{2}, \alpha^{2}\right)$ on the relative manifold $Y_{2} \stackrel{j_{2}}{\rightarrow} X_{2}$. We define a pullback of relative bundles along the morphism

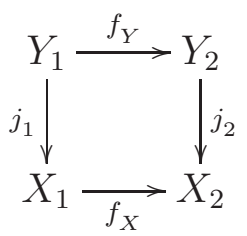

of relative manifolds. Since induction and pullback commute by remark 2.3(ii), we have a canonical isomorphism

$$
\operatorname{Ind}_{\iota}\left(f_{Y}^{*} P_{H}^{2}\right) \cong f_{Y}^{*} \operatorname{Ind}_{\iota} P_{H}^{2}
$$

of bundles. Noting that $f_{X} \circ j_{1}=j_{2} \circ f_{Y}$, we also have another isomorphism

$$
j_{1}^{*} f_{X}^{*} P_{G}^{2} \cong f_{Y}^{*} j_{2}^{*} P_{G}^{2}
$$

of $G$-bundles, and thus an isomorphism

$$
f_{Y}^{*}(\alpha): \quad \operatorname{Ind}_{\iota}\left(f_{Y}^{*} P_{H}^{2}\right) \rightarrow f_{Y}^{*} \operatorname{Ind}_{\iota} P_{H}^{2} \rightarrow f_{Y}^{*} f_{2}^{*} P_{G}^{2} \rightarrow j_{1}^{*} f_{X}^{*} P_{G}^{2}
$$


of $G$-bundles on $Y_{1}$. Hence $\left(f_{X}^{*} P_{G}^{2}, f_{Y}^{*} P_{H}^{2}, f_{Y}^{*}(\alpha)\right)$ is a relative $(G, H)$-bundle on $\left(X_{1}, Y_{1}\right)$.

We have thus a bifunctor $\operatorname{Bun}_{\iota: H \rightarrow G}$ from the category opposite to the category of relative manifolds to the bicategory of groupoids, i.e. a prestack $\operatorname{Bun}_{(G, H)}$ on the category of relative manifolds.

It should be appreciated that we do not require the group homomorphism $\iota: H \rightarrow G$ to be injective. For later use, we will consider two examples.

\section{Example 2.6.}

Consider the case that $X=Y$ is a point. Bundles are then torsors $\underline{H}$ and $\underline{G}$, respectively, which are unique up to isomorphism. The additional datum characterizing a relative bundle is then an isomorphism

$$
\alpha: \quad \underline{H} \times{ }_{H} G \stackrel{\cong}{\longrightarrow} \underline{G}
$$

of torsors. If we fix base points $*_{H} \in \underline{H}$ and $*_{G} \in \underline{G}$, then $\alpha$ is determined by the group element $\gamma_{\alpha} \in G$ such that $\alpha\left(\left[*_{H}, e\right]\right)=*_{G} \cdot \gamma_{\alpha}$.

Morphisms $(\underline{G}, \underline{H}, \alpha) \rightarrow\left(\underline{G}^{\prime}, \underline{H}^{\prime}, \alpha^{\prime}\right)$ are pairs of morphisms $\varphi_{H}: \underline{H} \rightarrow \underline{H^{\prime}}$ and $\varphi_{G}: \underline{G} \rightarrow \underline{G^{\prime}}$ of torsors. Using the base points $*_{H}$ and $*_{H}^{\prime}$ of $\underline{H}$ and $\underline{H}^{\prime}$, respectively, and similarly base points of the $G$-torsors, morphisms are described by group elements $g \in G$ and $h \in H$ such that

$$
\varphi_{H}\left(*_{H}\right)=*_{H}^{\prime} \cdot h \quad \text { and } \quad \varphi_{G}\left(*_{G}\right)=*_{G}^{\prime} \cdot g .
$$

The commuting diagram (2.26) requires that

$$
\varphi_{G}\left(\alpha\left[*_{H}, e\right]\right)=\varphi_{G}\left(*_{G} \cdot \gamma_{\alpha}\right)=*_{G}^{\prime} \cdot\left(g \gamma_{\alpha}\right)
$$

equals

$$
\alpha^{\prime}\left(\operatorname{Ind}_{\iota} \varphi_{H}\left(\left[*_{H}, e\right]\right)=\alpha^{\prime}\left(\left[*_{H}^{\prime} h, e\right]\right)=\alpha^{\prime}\left(\left[*_{H}^{\prime}, \iota(h)\right]\right)=*_{G}^{\prime} \cdot\left(\gamma_{\alpha^{\prime}} \iota(h)\right) .\right.
$$

We thus find the condition

$$
g \gamma_{\alpha}=\gamma_{\alpha^{\prime}} \iota(h)
$$

on the pair $(g, h)$ of group elements. As expected, for $\iota$ injective, this determines $h$ in terms of $g$. Moreover, given any two relative bundles, we can always find group elements $g$ and $h$ such that this relation holds. So there is a single isomorphism class of objects. In particular, we can restrict our attention to just one $H$-torsor $\underline{H}$ and one $G$-torsor $\underline{G}$. Then we get a category with objects labeled by $\gamma_{\alpha} \in G$ and morphisms being pairs $(g, h)$ such that $g \gamma_{\alpha}=\gamma_{\alpha^{\prime}} \iota(h)$, or put differently, the action groupoid

$$
G \backslash \backslash G / \iota^{-} H
$$

Here the notation is as follows. We deal with left actions for both $G$ and $H$. The left action of the group $G$ is simply left multiplication, while the left action of $H$ is right multiplication after applying the group homomorphism $\iota$ and taking the inverse, i.e. $(g, h) \cdot \gamma=g \cdot \gamma \cdot \iota(h)^{-1}$.

\section{Example 2.7.}

Take for $X$ a closed interval and for $Y$ the subset consisting of its two end points, which we label by 1,2. Since the interval is contractible and $G$ is finite, the category of $G$-bundles on $X$ is canonically equivalent to the category of $G$-torsors. Similarly we have $H_{1}$ - and $H_{2}$-torsors, one over each end point. We fix one such torsor for each end point and for the interval itself 
from now on. We also fix base points $*_{H_{1}}, *_{H_{2}}$ and $*_{G}$ for these torsors. Objects in the category are then pairs $\left(\gamma_{\alpha, 1}, \gamma_{\alpha, 2}\right) \in G \times G$ which describe the morphisms of torsors as

$$
\alpha_{1}\left(\left[*_{H_{1}}, e\right]\right)=*_{G} \cdot \gamma_{\alpha, 1} \quad \text { and } \quad \alpha_{2}\left(\left[*_{H_{2}}, e\right]\right)=*_{G} \cdot \gamma_{\alpha, 2} \cdot
$$

The morphisms are described by triples $\left(h_{1}, h_{2}, g\right) \in H_{1} \times H_{2} \times G$ satisfying

$$
\varphi_{H_{1}}\left(*_{H_{1}}\right)=*_{H_{1}} \cdot h_{1}, \quad \varphi_{H_{2}}\left(*_{H_{2}}\right)=*_{H_{2}} \cdot h_{2} \quad \text { and } \quad \varphi_{G}\left(*_{G}\right)=*_{G} \cdot g .
$$

Based on the commuting diagram (2.26), we check when a triple $\left(h_{1}, h_{2}, g\right)$ gives a morphism $\left(\gamma_{\alpha, 1}, \gamma_{\alpha, 2}\right) \rightarrow\left(\gamma_{\alpha, 1}^{\prime}, \gamma_{\alpha, 2}^{\prime}\right)$. As before we compute

$$
\varphi_{G}\left(\alpha_{i}\left[*_{H_{i}}, e\right]\right)=\varphi_{G}\left(*_{G} \gamma_{\alpha, i}\right)=*_{G} \cdot\left(g \gamma_{\alpha, i}\right)
$$

and

$$
\alpha_{i}^{\prime}\left(\operatorname{Ind}_{\iota} \varphi_{H}\left(\left[*_{H_{i}}, e\right]\right)=\alpha_{i}^{\prime}\left(\left[*_{H_{i}} h_{i}, e\right]\right)=\alpha^{\prime}\left(\left[*_{H_{i}}, \iota\left(h_{i}\right)\right]\right)=*_{G} \cdot\left(\gamma_{\alpha, i}^{\prime} \cdot \iota\left(h_{i}\right)\right) .\right.
$$

We thus arrive at the equalities

$$
g \gamma_{\alpha, i}=\gamma_{\alpha, i}^{\prime} \iota\left(h_{i}\right)
$$

for $i=1,2$. Hence the action groupoid is

$$
G \backslash \backslash \times G / /_{\iota_{1}^{-} \times \iota_{2}^{-}} H_{1} \times H_{2},
$$

where the $G$-action is the diagonal one.

\subsection{Groupoid cohomology and gerbes on groupoids}

The definition of a Dijkgraaf-Witten theory on a three-manifold requires, as an additional datum besides a finite group $G$, the choice of a 3-cocycle $\omega \in Z\left(G, \mathbb{C}^{\times}\right)$. This cocycle enters in the linearization. We now describe how this 3-cocycle can be seen geometrically as a 2-gerbe on the groupoid $* / / G$.

We first give a brief outline of groupoid cohomology. Given a finite groupoid $\Gamma=\left(\Gamma_{0}, \Gamma_{1}\right)$, consider its nerve, which is a simplicial set

$$
\left(\cdots \underset{\partial_{3}}{\stackrel{\partial_{0}}{\rightleftharpoons}} \Gamma_{2} \underset{\partial_{2}}{\stackrel{\partial_{0}}{\rightleftarrows}} \Gamma_{1} \underset{\partial_{1}}{\stackrel{\partial_{0}}{\rightleftarrows}} \Gamma_{0}\right)=: \Gamma_{\bullet},
$$

where for $i \geq 1, \Gamma_{i}$ consists of $i$-tuples of composable morphisms of $\Gamma$. Applying the functor $\operatorname{Map}\left(-, \mathbb{C}^{\times}\right)$and taking alternating combinations of the face maps yields a complex

$$
\operatorname{Map}\left(\Gamma_{0}, \mathbb{C}^{\times}\right) \rightarrow \operatorname{Map}\left(\Gamma_{1}, \mathbb{C}^{\times}\right) \rightarrow \operatorname{Map}\left(\Gamma_{2}, \mathbb{C}^{\times}\right) \rightarrow \operatorname{Map}\left(\Gamma_{3}, \mathbb{C}^{\times}\right) \rightarrow \cdots
$$

of groups. A group $G$ gives rise to the groupoid $* / / G$ with a single object. In this case the complex (2.44) reduces to the standard bar complex.

It is useful to think about cochains in this complex in a geometric way.

\section{Definition 2.8.}

An $n$-gerbe on the groupoid $\Gamma$ is an $(n+1)$-cocycle

$$
\omega \in Z^{n+1}\left(\Gamma, \mathbb{C}^{\times}\right) .
$$


Using standard facts about complexes in small abelian categories one deduces that $n$-gerbes on a groupoid $\Gamma$ form an $n+1$-category:

- A (-1)-gerbe is an object in degree 0, i.e. an element of the set of objects of $\Gamma$.

- A 0 -gerbe consists of a 1-cocycle $\omega \in Z^{1}(\Gamma)$. The morphism sets are

$$
\operatorname{Hom}\left(\omega, \omega^{\prime}\right)=\left\{\eta \in \Gamma_{0} \mid \mathrm{d} \eta=\omega^{\prime}-\omega\right\}
$$

We thus get a category of 0 -gerbes, which we also call line bundles on $\Gamma$. Its isomorphism classes are classified by the cohomology group $H^{1}\left(\Gamma, \mathbb{C}^{\times}\right)$.

- 1-gerbes form a bicategory. Its objects are 2-cocycles, and the set of 1-morphisms between two 2-cocycles $\omega$ and $\omega^{\prime}$ is $\left\{\eta \in \Gamma_{1} \mid \mathrm{d} \eta=\omega^{\prime}-\omega\right\}$. Given two 1-morphisms $\eta, \eta^{\prime}: \omega \rightarrow \omega^{\prime}$, a 2-morphism $\Phi: \eta \Rightarrow \eta^{\prime}$ is an element $\Phi \in \Gamma_{0}$ satisfying $\mathrm{d} \Phi=\eta^{\prime}-\eta$.

The isomorphism classes of this bicategory of gerbes are classified by the cohomology group $H^{2}\left(\Gamma, \mathbb{C}^{\times}\right)$.

For Dijkgraaf-Witten theories based on a finite group $G, 2$-gerbes on the groupoid $* / / G$ are relevant. As we already have pointed out, they should be thought of as a finite version of a Chern-Simons 2-gerbe.

\subsection{Module categories over the fusion category $G$-vect ${ }^{\omega}$}

We next discuss category-theoretic and algebraic realizations of group 3-cocycles. A closed 3 -cocycle $\omega$ on a finite group $G$ allows one to endow the abelian category $G$-vect of $G$-graded vector spaces with a non-trivial associativity constraint, defined on simple objects by

$$
\begin{aligned}
a_{V_{g_{1}}, V_{g_{2}}, V_{g_{3}}}: \quad\left(V_{g_{1}} \otimes V_{g_{2}}\right) \otimes V_{g_{3}} & \rightarrow V_{g_{1}} \otimes\left(V_{g_{2}} \otimes V_{g_{3}}\right) \\
v_{1} \otimes v_{2} \otimes v_{3} & \mapsto \omega\left(g_{1}, g_{2}, g_{3}\right) v_{1} \otimes v_{2} \otimes v_{3} .
\end{aligned}
$$

This yields a fusion category, which is denoted by $G$-vect ${ }^{\omega}$ (the pentagon axiom is fulfilled because $\omega$ is closed). Cohomologous 3-cocycles give rise to monoidally equivalent fusion categories.

The modular tensor category relevant for the Dijkgraaf-Witten theory based on $(G, \omega)$ is the Drinfeld center $\mathcal{Z}\left(G\right.$-vect $\left.^{\omega}\right)$. (This has been discussed in DPR; a helpful more recent exposition is given in [Wi].) It is thus a topological field theory of Reshetikhin-Turaev type. This allows us to compare our geometric results with those obtained in the model independent approach to defects and boundary conditions in [FSV].

The indecomposable module categories over the monoidal category $G$-vect ${ }^{\omega}$ have been classified [Os2, Example 2.1]: Consider a subgroup $H \leq G$ and a 2-cochain $\theta$ on $H$ such that $\mathrm{d} \theta=\left.\omega\right|_{H}$. Note that this requires the restriction of $\omega$ to the subgroup $H$ to be exact and thus imposes in general restrictions on the subgroup. Rephrased in the language of Section 2.4, $\theta$ is a 1-morphism from the trivial 2-gerbe on $* / / H$ to the pullback 2-gerbe $\iota^{*} \omega$.

The twisted group algebra $A_{H, \theta}:=\mathbb{C}_{\theta}[H]$ is then a (haploid special symmetric) Frobenius algebra in $G$-vect ${ }^{\omega}$. For any 1-cochain $\chi$ on $H$ the algebras $A_{H, \theta}$ and $A_{H, \theta+d \chi}$ are isomorphic. Thus, given a subgroup $H$ the isomorphism classes of algebras form a torsor over $H^{2}\left(H, \mathbb{C}^{\times}\right)$. Indecomposable module categories over $G$-vect ${ }^{\omega}$ are given by Morita classes of twisted group 
algebras. They are thus in bijection with equivalence classes of pairs $(H, \theta)$; we denote them by $\mathcal{M}_{H, \theta}$.

Actually, any pair consisting of a group homomorphism $\iota: H \rightarrow G$ and a 2-cochain $\theta$ on $H$ such that $\iota^{*} \omega=\mathrm{d} \theta$ defines a module category, albeit not an indecomposable one unless $\iota$ is injective. For the case that both $\omega$ and $\theta$ vanish, this is discussed in the Appendix.

\section{Categories of generalized Wilson lines in Dijkgraaf- Witten theories}

We are now ready to discuss Dijkgraaf-Witten theories with boundaries and defects. Our ultimate goal is to consider such a theory as a 1-2-3-extended topological field theory. Concretely this means:

- To a decorated smooth oriented one-dimensional manifold, we have to assign a finitely semisimple $\mathbb{C}$-linear category.

This category will have the interpretation of a category of (generalized) Wilson lines. The one-dimensional manifold is allowed to have boundaries, corresponding to physical boundaries of the three-dimensional theories, and to have marked points, corresponding to surface defects.

- To a decorated smooth oriented two-dimensional manifold we have to assign a $\mathbb{C}$-linear functor. A two-dimensional manifold can have physical boundaries and lines corresponding to surface defects. Moreover, it can have cut-and-paste boundaries which are one-dimensional manifolds of the type described in the first item. These cut-and-paste boundaries determine the categories which are the source and target for the functor associated to the two-manifold.

- To a decorated three-manifold with corners, we have to associate a natural transformation.

\subsection{Decorated one-manifolds and categories of generalized bundles}

In the present paper we concentrate on examples and restrict our attention to one-dimensional manifolds. We should also keep in mind that cut-and-paste boundaries have been introduced to implement locality. Accordingly we impose the condition that a cutting is transversal to any additional decoration data such as surface defects or generalized Wilson lines.

This leaves us with two types of connected one-manifolds only:

- An interval which is partitioned by finitely many distinct points in its interior.

- A circle that is partitioned by finitely many distinct points.

For the situations shown in (2.11) above, the cutting leading to such one-manifolds is indicated 
in the following picture:
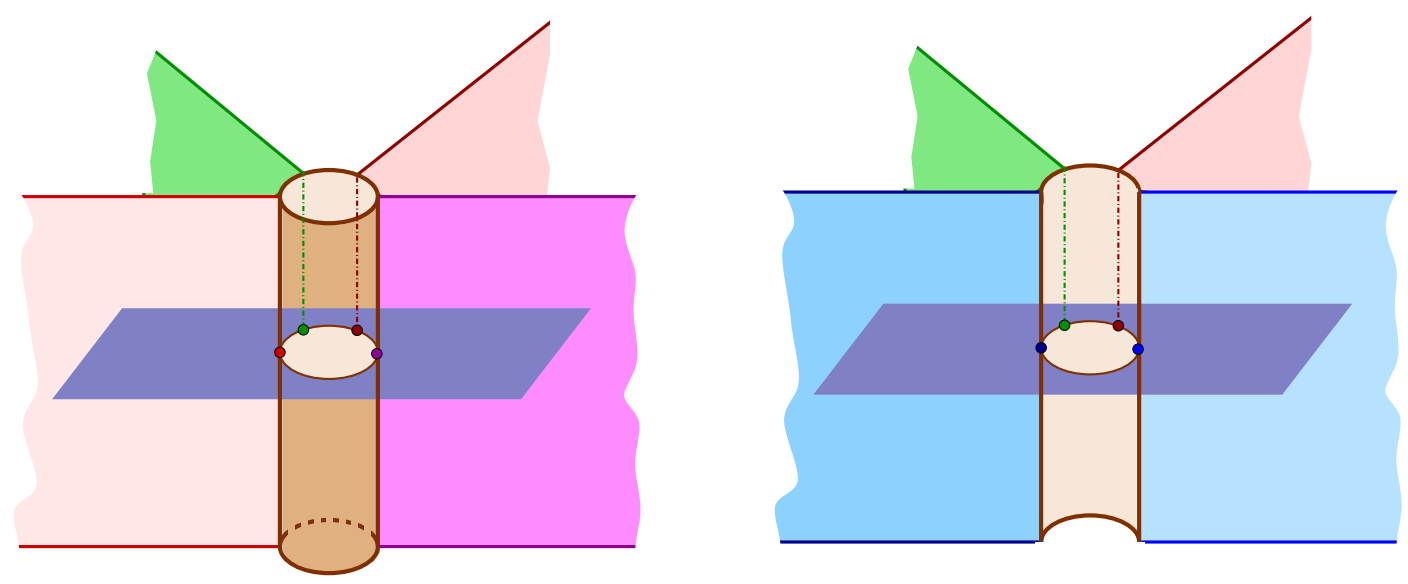

Every subinterval of such a one-manifold is decorated by a Dijkgraaf-Witten theory. The decoration datum for each subinterval is thus a finite group $G$ together with a 3-cocycle $\omega \in Z^{3}\left(G, \mathbb{C}^{\times}\right)$. The locality of the geometric construction of Dijkgraaf-Witten theories [Fr, Mor] then suggests that $G$-bundles on these intervals should appear in our construction.

However, we also must assign data to the end points of a subinterval. Recall from Section 2.1 that the general construction of Dijkgraaf-Witten theories consists of two steps: first finding an appropriate stack of bundles, leading to spans of groupoids, which then have to be linearized with the help of Lagrangian data. In the situation at hand, the relevant categories are variants of relative bundles which have been introduced in Section 2.3. In the case of an interval without marked points in the interior, the morphism defining the relative manifold is the embedding of the end points.

One might thus pick a group homomorphism $\iota: H \rightarrow G$ and assign $H$-bundles to the two end points. This is, however, not the most general situation one can consider - for complying with locality we must allow for the possibility to assign different local conditions to the two end points of the interval. Thus we select possibly different groups $H_{i}, i=1,2$, and group homomorphism $\iota_{i}: H_{i} \rightarrow G$ separately for each end point $p_{1}, p_{2}$ and consider the following category: an object consists of a $G$-bundle $P_{G}$ over the interval, an $H_{1}$-bundle $P_{H_{1}}$ over $p_{1}$, a morphism $\left.\operatorname{Ind}_{\iota_{1}} P_{H_{1}} \rightarrow\left(P_{G}\right)\right|_{p_{1}}$ of $G$-bundles on $p_{1}$, an $H_{2}$-bundle $P_{H_{2}}$ over $p_{2}$, and a morphism $\left.\operatorname{Ind}_{\iota_{2}} P_{H_{2}} \rightarrow\left(P_{G}\right)\right|_{p_{2}}$ of $G$-bundles on $p_{2}$.

This leads to the following assignment of kinematical data. At the level of groups, we associate to an end point of an interval that is labeled by a group $G$ a group homomorphism $\iota: H \rightarrow G$, with $H$ some finite group. This prescription still needs to be complemented by group cohomological Lagrangian data; these will be introduced in Section 3.2 .

Example 2.7 allows us to determine directly a finite action groupoid that is relevant for an interval without any marked interior points, labeled by a group $G$, and with end points labeled by groups $H_{1}, H_{2}$ and group homomorphisms $\iota_{1}: H_{1} \rightarrow G$ and $\iota_{2}: H_{2} \rightarrow G$ respectively: it is given by

$$
G \backslash G \times G / /_{\iota_{1}^{-} \times \iota_{2}^{-}} H_{1} \times H_{2} .
$$

Here $G$ acts from the left as the diagonal subgroup, while $H_{1}$ is mapped via $\iota_{1}$ to the first copy of $G$ and acts by right multiplication after taking the inverse; the action of $H_{2}$ is analogous, the only difference being that it is mapped by $\iota_{2}$ into the second copy of $G$. Let us describe 
the structure of this groupoid: its set of objects is given by a Cartesian product of groups, one factor for each pair consisting of a marked point and a neighbouring interval. The group is determined by the interval, since it comes from the morphism of bundles in the corresponding relative bundle. The morphisms in the groupoid are gauge transformations: the $G$-action describes gauge transformations of the $G$-bundle on the interval and acts by multiplication from the left. The $H_{i}$-actions are by multiplication from the right after having taken the inverse; their origin are $H_{i}$-gauge transformations of the $H_{1}$-bundles on the respective marked point.

This picture generalizes to marked points in the interior, either of an interval or of a circle. To any such point two intervals are adjacent, which are labeled by gauge groups $G_{1}$ and $G_{\mathrm{r}}$, respectively. To describe the resulting relative manifold, consider as an example the closed interval $[0,1]$ with a marked interior point $p_{1}:=\frac{1}{2}$. Take for $X$ the disjoint union $X:=\left[0, \frac{1}{2}\right] \sqcup\left[\frac{1}{2}, 1\right]$. One should appreciate that in $X$ the point $p_{1}$ is "doubled". By locality, the category of bundles

is now defined with separate data for each of the marked points $p_{0}=0, p_{1}=\frac{1}{2}$ and $p_{2}=1$. For $p_{0}$ and $p_{2}$ we select again group homomorphisms $\iota_{0}: H_{0} \rightarrow G_{1}$ and $\iota_{2}: H_{2} \rightarrow G_{\mathrm{r}}$. At $p_{1}$ we take as a datum a finite group $H_{1}$ and a group homomorphism $\iota: H_{1} \rightarrow G_{1} \times G_{\mathrm{r}}$ or, equivalently, a pair of group homomorphisms $\iota_{1}: H_{1} \rightarrow G_{1}$ and $\iota_{\mathrm{r}}: H_{2} \rightarrow G_{\mathrm{r}}$.

We consider thus for a given one-manifold $S$ the following geometric category: an object is the assignment of a $G$-bundle to each subinterval labeled by a finite group $G$ and of $H$-bundles to marked points in the interior or end points. The final datum are compatible morphisms from induced bundles to restrictions of bundles at all marked points. We denote this geometric category by $\operatorname{Bun}(S)$.

\section{Definition 3.1.}

(i) A one-dimensional pre- $D W$ manifold is a smooth one-dimensional manifold $S$, possibly with boundary, together with the following data:

- A finite set $P_{S}$ of points of $S$, containing all boundary points of $S$.

We refer to the elements of $P_{S}$ as marked points, and to a connected component of $S \backslash P_{S}$ as a subinterval of $S$. We choose an orientation for each subinterval.

- To each subinterval of $S$ we associate a finite group.

- To a marked point $p \in P_{S}$ that is a boundary point and is thus adjacent to a single subinterval $I$ with associated group $G$, we select a finite group $H$ and a group homomorphism $\iota: H \rightarrow G$. To a marked point $p \in P_{S}$ that is not a boundary point of $S$ and is thus adjacent to two subintervals $I_{1}$ and $I_{2}$, labeled by finite groups $G_{1}$ and $G_{2}$, respectively, we select a finite group $H$ and a pair of group homomorphisms $\iota_{i}: H \rightarrow G_{i}$.

(ii) To a one-dimensional pre-DW manifold $S$, we associate the category $\operatorname{Bun}(S)$ of bundles described above. This is an essentially finite groupoid.

(iii) Each subinterval of a one-dimensional pre-DW manifold $S$ is endowed with an orientation. Thereby any marked point $p \in P_{S}$ is either a start point or an end point for any interval $I$ adjacent to $p$. In the first case, we set $\epsilon(p, I):=+1$, in the latter $\epsilon(p, I):=-1$.

To make contact with the results in [FSV] which use the theory of module categories, we need to find finite groupoids that are equivalent to groupoids $\operatorname{Bun}(S)$ of relative bundles of pre-DW manifolds. This is the goal of the remaining part of this subsection. 
As a first example, consider a circle with one marked point, which corresponds to a surface defect. If we associate to the interval the group $G$, then we have to associate to the defect a group homomorphism $\iota: H \rightarrow G \times G$, and the resulting action groupoid is

$$
G \backslash \backslash G \times G / \iota^{-} H
$$

Of particular interest is the case that the group homomorphism $\iota$ is the embedding homomorphism of the diagonal subgroup $G \leq G \times G$. We denote by $G / /_{\text {ad }} G$ the action groupoid for the left adjoint action of $G$ on itself. The functor

$$
F: \quad G \backslash \backslash G \times G / / G \rightarrow G / /_{\mathrm{ad}} G
$$

that acts on objects as $F\left(\gamma_{1}, \gamma_{2}\right)=\gamma_{1} \gamma_{2}^{-1}$ and on morphisms as

$$
F\left(\left(\gamma_{1}, \gamma_{2}\right) \stackrel{\left(h_{1}, h_{2}\right)}{\longrightarrow}\left(h_{1} \gamma_{1} h_{2}^{-1}, h_{1} \gamma_{2} h_{2}^{-1}\right)\right)=\left(\gamma_{1} \gamma_{2}^{-1} \stackrel{h_{1}}{\longrightarrow} h_{1} \gamma_{1} \gamma_{2}^{-1} h_{1}^{-1}\right)
$$

is an equivalence of categories. We will see that the linearization of the adjoint action groupoid together with the relevant cocycle (see formula (3.46) ) produces the appropriate category associated to the circle without marked points, i.e. the category of ordinary bulk Wilson lines.

As a more involved example, let us discuss a circle with two marked points. We describe the circle as $S^{1}=\{z \in \mathbb{C}|| z \mid=1\}$ and take the marked points to be $\pm \mathrm{i} \in S^{1}$. For the two intervals that consist of points with positive and negative real parts, respectively, we choose groups $G_{>}$ and $G_{<}$, respectively. At the points \pm i, we choose group homomorphisms

$$
\iota_{+}: \quad H_{+} \rightarrow G_{>} \times G_{<} \quad \text { and } \quad \iota_{-}: \quad H_{-} \rightarrow G_{<} \times G_{>} .
$$

The relevant action groupoid is then

$$
G_{>} \times G_{<} \backslash \backslash G_{>} \times G_{<} \times G_{<} \times G_{>} / /_{\iota_{+}^{-} \times \iota_{-}^{-}} H_{+} \times H_{-},
$$

where the action of $G_{>}$and $G_{<}$is again diagonal and the left action of $H_{ \pm}$is again by right multiplication preceded by applying the relevant group homomorphism and taking inverses. This description generalizes in an obvious manner to circles with an arbitrary finite number of marked points. The generalization to intervals with an arbitrary finite number of marked points is easy as well. We have thus succeeded in describing for a specific type of one-dimensional pre-DW manifold the category $\operatorname{Bun}(S)$ by a finite action groupoid.

We discuss again a specific case: suppose that $G_{>}=G_{<}=: G$ and that $H_{+} \cong G \stackrel{d}{\rightarrow} G \times G$ is the diagonal subgroup, while $\iota_{-}=\iota: H \rightarrow G \times G$ is an arbitrary group homomorphism. Then the relevant action groupoid is

$$
G \times G \backslash \backslash G \times G \times G \times G / /_{d^{-} \times \iota^{-}} G \times H
$$

with the first copy of $G$ in the gauge group $G \times G$ acting on the first and forth copies of $G$ in $G \times G \times G \times G$ by left multiplication and the second copy of $G$ acting on the second and third copies. The left action of $G$ on the right is as a subgroup of the first and second copy of $G$. The action groupoid (3.8) is equivalent to the action groupoid

$$
G \backslash \backslash G \times G / /_{\iota^{-}} H
$$


via the functor $F$ that acts on objects as

$$
F\left(\gamma_{1}, \gamma_{2}, \gamma_{3}, \gamma_{4}\right):=\left(\gamma_{1} \gamma_{2}^{-1} \gamma_{3}, \gamma_{4}\right)
$$

and maps the morphism

$$
\left(\gamma_{1}, \gamma_{2}, \gamma_{3}, \gamma_{4}\right) \stackrel{\left(g_{1}, g_{2}, g, h\right)}{\longrightarrow}\left(g_{1} \gamma_{1} g^{-1}, g_{2} \gamma_{2} g^{-1}, g_{2} \gamma_{3} h^{-1}, g_{1} \gamma_{4} h^{-1}\right)
$$

in the groupoid (3.8) to the morphism

$$
\left(\gamma_{1} \gamma_{2}^{-1} \gamma_{3}, \gamma_{4}\right) \stackrel{\left(g_{1}, h\right)}{\longrightarrow}\left(g_{1} \gamma_{1} \gamma_{2}^{-1} \gamma_{3} h^{-1}, g_{1} \gamma_{4} h^{-1}\right)
$$

in (3.9). It is straightforward to check that this functor is surjective and a bijection on morphism spaces and is thus an equivalence of groupoids.

\subsection{Lagrangian data and linearization of groupoids}

We now proceed to the linearization process. This requires additional data which come from the cohomology of the groupoids that have to be linearized. These data have the physical interpretation of (topological) Lagrangians and appropriate boundary terms.

We introduce such additional data as follows. To an end point of an interval that is adjacent to a subinterval labeled by a finite group $G$ and 3-cocycle $\omega$ we associate a group homomorphism $\iota: H \rightarrow G$ and a 2-cochain $\theta \in C^{2}\left(H, \mathbb{C}^{\times}\right)$such that $\mathrm{d} \theta=\iota^{*} \omega$. It is appropriate to think about $\theta$ as a morphism triv $\rightarrow \iota^{*} \omega$ of 2 -gerbes on the groupoid $* / / H$. The situation can be regarded as a higher categorical analogue of the role played by gerbe modules in the description of boundary conditions in two-dimensional theories with non-trivial Wess-Zumino terms (see e.g. [FNSW, Sect.6] for an exposition using gerbes and gerbe modules). In the two-dimensional situation, one has a gerbe module on a submanifold $\iota: \Sigma \rightarrow M$, which amounts to a 1-morphism $I_{\omega} \rightarrow \iota^{*} \mathcal{G}$ of gerbes on $\Sigma$ from a trivial gerbe $I_{\omega}$ to the restriction of the gerbe $\mathcal{G}$ on $M$. In the present situation we have a module of a 2-gerbe; technical simplifications come from the fact that the groups we deal with are finite and that thus any infinitesimal data related to connections are trivial.

In the case of two intervals adjacent to one another, labeled by $\left(G_{1}, \omega_{1}\right)$ and $\left(G_{2}, \omega_{2}\right)$, respectively, we choose a group homomorphism $\iota=\left(\iota_{1}, \iota_{2}\right): H \rightarrow G_{1} \times G_{2}$ and a 2-cochain $\theta$ on $H$ such that $d \theta=\left(\iota_{2}^{*} \omega_{2}\right) \cdot\left(\iota_{1}^{*} \omega_{1}\right)^{-1}$. Again the situation has an analogue in two dimensions: defects in backgrounds with non-trivial Wess-Zumino term are described by gerbe bimodules and bibranes, see [FSW] and [FNSW, Sect. 7] for a review.

We summarize these prescriptions in the following

Definition 3.2. A one-dimensional $D W$ manifold is a one-dimensional pre-DW manifold $S$ together with the following choice of Lagrangian data:

- To each subinterval of $S$ with finite group $G$, we associate a closed 3-cochain on $G$.

- To a marked boundary point $p \in P_{S} \cap \partial S$ adjacent to a subinterval with group $G$ and 3-cocycle $\omega \in Z^{3}\left(G, \mathbb{C}^{\times}\right)$and labeled with a group homomorphism $\iota: H \rightarrow G$, we assign a 2-cochain $\theta \in C^{2}\left(H, \mathbb{C}^{\times}\right)$such that

$$
\mathrm{d} \theta=\iota^{*} \omega^{\epsilon(p, I)},
$$

with $\epsilon(p, I)$ as defined in Definition 3.1(iii). 
- To a marked interior point $p \in P_{S} \backslash \partial S$ adjacent to subintervals $I_{1}$ and $I_{2}$ with group homomorphisms $\iota_{i}: H \rightarrow G_{i}$ we assign a cochain $\theta \in C^{2}\left(H, \mathbb{C}^{\times}\right)$such that

$$
\mathrm{d} \theta=\iota_{1}^{*} \omega_{1}^{\epsilon\left(p, I_{1}\right)} \cdot \iota_{2}^{*} \omega_{2}^{\epsilon\left(p, I_{2}\right)} .
$$

We now use the data of a DW manifold to define twisted linearizations of the groupoids that we constructed in the previous subsection. Let us describe the general idea of a twisted linearization of a finite groupoid $H \backslash G$ given by a left action of a group $H$ on a set $G$. The ordinary linearization is the functor category $[H \backslash G$, vect $\mathbb{C}]$. An object of this category is given by

- A finite-dimensional vector space $V_{\gamma}$ for each element $\gamma \in G$.

- For each $\gamma \in G$ and $h \in H$ a linear map $\rho_{h}: V_{\gamma} \rightarrow V_{h . \gamma}$ such that the diagram

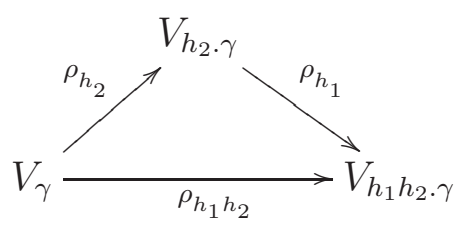

commutes for all $\gamma \in G$ and $h_{1}, h_{2} \in H$.

Morphisms in the functor category are natural transformations; explicitly, they are $G$-homogeneous maps commuting with the $H$-action.

The additional input datum for a twisted linearization is a 2-cocycle $\tau$ on the groupoid $H \backslash \backslash G$. This gives rise to the following twisted version of the functor category $[H \backslash \backslash G$, vect $\mathbb{C}]$ (see also [Mor, Sect. 5.4]):

Definition 3.3. The $\tau$-twisted linearization of the groupoid $H \backslash \backslash G$, denoted by $[H \backslash G \text {, vect }]^{\tau}$, is the following category. An object of $[H \backslash G \text {, vect } \mathbb{C}]^{\tau}$ consists of

- A finite-dimensional vector space $V_{\gamma}$ for each $\gamma \in G$.

- For each $h \in H$ a linear map $\rho_{h}: V_{\gamma} \rightarrow V_{h . \gamma}$ such that the composition law of the $H$-action is realized projectively, i.e. up to the scalar factor $\tau\left(h_{1}, h_{2} ; \gamma\right) \in \mathbb{C}^{\times}$. Diagrammatically,

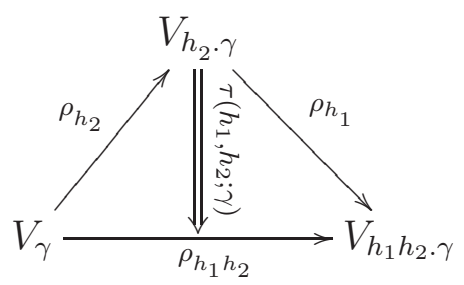

As a formula,

$$
\rho_{h_{1} h_{2}}=\tau\left(h_{1}, h_{2} ; \gamma\right) \rho_{h_{1}} \rho_{h_{2}} .
$$

Morphisms of $[H \backslash G \text {, vect }]_{\mathbb{C}}^{\tau}$ are $G$-homogeneous maps commuting with the $H$-action. 


\subsection{2-cocycles from Lagrangian data}

Our next task is thus to use the Lagrangian data that are part of the data of a one-dimensional DW-manifold. We have assigned them in Definition 3.2 to intervals and circles with marked points to produce 2-cocycles for the groupoids discussed in Section 3.1. For brevity we consider in this subsection Lagrangian data for boundaries only; the discussion for surface defects is similar.

Any homomorphism $\iota: H \rightarrow G$ of finite groups provides a morphism $\iota: B H \rightarrow B G$ of the corresponding classifying spaces. Assume now that we are given a 3-cocycle $\omega \in Z^{3}\left(B G, \mathbb{C}^{\times}\right)$ and a 2-cochain $\theta \in C^{2}\left(B H, \mathbb{C}^{\times}\right)$such that

$$
i^{*} \omega=\mathrm{d} \theta .
$$

We recall that a $G$-bundle on a manifold $M$ can be described by a map from $M$ to the classifying space $B G$. Morphisms of bundles can be described by homotopies between such maps. Thus for $\Sigma$ an oriented one-dimensional manifold with boundary, a relative bundle on the relative manifold $(\Sigma, \partial \Sigma)$ leads to the following data (up to homotopy):

- A map $f \in \operatorname{Map}(\Sigma, B G)$ describing a $G$-bundle on $\Sigma$.

- A map $g \in \operatorname{Map}(\partial \Sigma, B H)$ describing an $H$-bundle on $\partial \Sigma$.

- A homotopy describing the morphism of bundles, i.e. a map $h \in \operatorname{Map}([0,1], \operatorname{Map}(\partial \Sigma, B G))$, with $[0,1]$ the standard interval.

We will later need the subset $X_{\circ}$ consisting of such triples $(f, g, h)$ subject to the condition that $h$ is a homotopy relating the maps $\left.f\right|_{\partial \Sigma}$ and $\iota \circ g$ from $\partial \Sigma$ to $B G$,

$$
X_{\circ}:=\left\{(f, g, h)|f|_{\partial \Sigma} \simeq^{h} i \circ g\right\} .
$$

Each point of $X_{\circ}$ describes a relative bundle, i.e. an object of $\operatorname{Bun}_{(G, H)}(\partial \Sigma \rightarrow \Sigma)$. Isomorphism classes of relative bundles are in bijection with the set $\pi_{0}\left(X_{\circ}\right)$ of connected components of $X_{\circ}$.

From the cohomological data $\omega$ and $\theta$ we now build a 2-cocycle in $Z^{2}\left(X_{\circ}, \mathbb{C}^{\times}\right)$. To this end we use the evaluation map

$$
\text { ev : } \quad \Sigma \times \operatorname{Map}(\Sigma, B G) \rightarrow B G
$$

to define a cochain $\tau_{\Sigma}(\omega) \in C^{2}\left(\operatorname{Map}(\Sigma, B G), \mathbb{C}^{\times}\right)$by

$$
\tau_{\Sigma}(\omega):=\int_{\Sigma} \operatorname{ev}^{*} \omega
$$

where $\int_{\Sigma}$ denotes the pushforward along the fibration $p_{2}: \Sigma \times \operatorname{Map}(\Sigma, B G) \rightarrow \operatorname{Map}(\Sigma, B G)$. As $\Sigma$ can have a non-empty boundary, there is, in general, no reason that the cochain $\tau_{\Sigma}(\omega)$ should be closed.

By the same procedure we obtain a 2-cochain $\left.\tau_{\partial \Sigma}(\theta) \in C^{2}(\operatorname{Map}(\partial \Sigma, B H)), \mathbb{C}^{\times}\right)$, as well as a 2-cochain $\tau_{[0,1]}\left(\tau_{\partial \Sigma}(\omega)\right) \in C^{2}\left(\operatorname{Map}([0,1], \operatorname{Map}(\partial \Sigma, B G)), \mathbb{C}^{\times}\right)$. We then consider the product space

$$
X:=\operatorname{Map}(\Sigma, B G) \times \operatorname{Map}(\partial \Sigma, B H) \times \operatorname{Map}([0,1], \operatorname{Map}(\partial \Sigma, B G)) .
$$

The pullbacks along the canonical projections $p_{i}$ to the three factors of (3.22) supply us with a 2-cochain on $X$ :

$$
\varphi:=p_{1}^{*} \tau_{\Sigma}(\omega)-p_{2}^{*} \tau_{\partial \Sigma}(\theta)-p_{3}^{*} \tau_{[0,1]}\left(\tau_{\partial \Sigma}(\omega)\right)
$$


The space $X_{\circ}$ introduced in (3.19) to describe relative bundles is by definition a subspace of $X$ (3.22). The central insight is now that the 2 -cochain that is obtained by restricting $\varphi$ to the subspace $X_{\circ}$ of $X$ is closed,

$$
\left.\mathrm{d} \varphi\right|_{X_{\circ}}=0 .
$$

In other words, we have obtained a 2-cocycle $\left.\varphi\right|_{X_{\circ}} \in Z^{2}\left(X_{\circ}, \mathbb{C}^{\times}\right)$on the space $X_{\circ}$ describing relative bundles.

To see that (3.24) holds, we work for the moment with differential forms and consider an arbitrary manifold $U$. Consider $\alpha \in \Omega_{\mathrm{cl}}^{3}(\Sigma \times U, \mathbb{R})$ and $\left.\beta \in \Omega^{2}(\partial \Sigma \times U), \mathbb{R}\right)$ obeying $\left.\alpha\right|_{\partial \Sigma \times U}=\mathrm{d} \beta$. Taking into account that $\Sigma$ has a boundary, we have

$$
\mathrm{d}\left(\int_{\Sigma} \alpha\right)=\int_{\Sigma} \mathrm{d} \alpha+\left.\int_{\partial \Sigma} \alpha\right|_{\partial \Sigma \times U}=\int_{\Sigma} \mathrm{d} \alpha+\int_{\partial \Sigma} \mathrm{d} \beta=\int_{\partial \Sigma} \mathrm{d} \beta .
$$

This means that the form

$$
\phi:=\int_{\Sigma} \alpha-\int_{\partial \Sigma} \beta \in \Omega^{2}(U, \mathbb{R})
$$

is closed, $\mathrm{d} \phi=0$. The same argument applies to elements in $Z^{3}\left(\Sigma \times U, \mathbb{C}^{\times}\right)$where slant products are used as the analogue of integration along the fiber.

The argument can now be applied to the situation of our interest: The role of $\int_{\Sigma} \alpha$ is then played by $\left.p_{1}^{*} \tau_{\Sigma}(\omega)\right|_{X_{\circ}}$ and the role of $\int_{\partial \Sigma} \beta$ by $\left.\left(p_{2}^{*} \tau_{\partial \Sigma}(\varphi)+p_{3}^{*} \tau_{[0,1]}\left(\tau_{\partial \Sigma}(\omega)\right)\right)\right|_{X_{\circ}}$. Their difference is precisely the combination $\varphi$ introduced in (3.23). From the relation $\left.\alpha\right|_{\partial \Sigma \times U}=\mathrm{d} \beta$ we thus obtain the desired equality (3.24).

\subsection{Graphical calculus for groupoid cocycles}

Generalizing the approach of [Wi], we can achieve a more combinatorial description of the 2cocycles on the groupoids derived in Section 3.1. We formulate it with the help of an algorithm which is based on three-dimensional diagrams and their decomposition into simplices. The diagrams are obtained from a graphical representation of the groupoids involved.

We start with a one-dimensional diagram, drawn vertically, which represents a one-dimensional pre-DW manifold to which we wish to associate a category by linearization. These manifolds are circles or intervals with finitely many marked points, including boundary points in the case of intervals. Each subinterval is marked by a finite group $G_{i}$ and a 3-cocycle $\omega_{i} \in Z^{3}\left(G_{i}, \mathbb{C}^{\times}\right)$. For each marked point we have a group $H_{j}$ and group homomorphisms to the groups associated with the adjacent intervals. The data characterizing an object in the associated groupoid described in Section 3.1 are then elements in the groups $G_{i}$ associated to the subinterval, one for each point adjacent to the subinterval.

Our convention is now to draw an empty circle for a marked point and to replace the original subintervals by filled circles. Between these circles we draw edges which are labeled by elements of the groups $G_{i}$ that are part of the data describing a relative bundle. An example is depicted 
in the following picture:

$$
\left\{\begin{array}{lll}
H_{1}, \iota_{1} & & \\
G_{1} & & \\
H_{12}, \iota_{12}^{1}, \iota_{12}^{2} & & \gamma_{3} \\
G_{2} & & \gamma_{4} \\
H_{23}, \iota_{23}^{2}, \iota_{23}^{3} & & \gamma_{5} \\
G_{3} & & \gamma_{6} \\
H_{3}, \iota_{3} & & \vdots
\end{array}\right.
$$

The figure on the left hand side of (3.27) shows the pre-DW-manifold $S$ which is an interval with two interior marked points, together with the relevant groups and group homomorphisms. The labels in the figure on the right hand side are group elements $\gamma_{1}, \gamma_{2} \in G_{1}, \gamma_{3}, \gamma_{4} \in G_{2}$ and $\gamma_{5}, \gamma_{6} \in G_{3}$ i that specify an object in $\operatorname{Bun}(S)$..

A morphism in the groupoid consists of elements of the groups $H_{j}$ and $G_{i}$ describing gauge transformations of the involved bundles. We represent such morphisms by two-dimensional diagrams with oriented edges as follows:

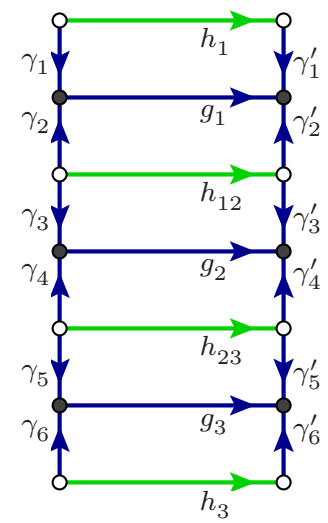

Here horizontal edges connecting empty circles are labeled by elements of the groups $H_{j}$, while horizontal edges connecting filled circles are labeled by elements of the groups $G_{i}$. For each square in the diagram there is a consistency condition relating the labels of its edges. To formulate this condition, we adopt the convention that orientation reversal amounts to inversion of the group element that labels the edge:

$$
\gamma \downarrow \quad \hat{=} \quad \gamma^{-1}
$$

With this convention the product of all group elements (possibly after applying an appropriate group homomorphism $H_{j} \rightarrow G_{i}$ ) along a closed curve equals the neutral element; we refer to this relation as the holonomy condition. For instance, the holonomy condition for the top square in (3.28) is the equality

$$
\gamma_{1}^{\prime} \cdot \iota_{1}\left(h_{1}\right)=g_{1} \cdot \gamma_{1}
$$


in $G_{1}$. This determines the element $\gamma_{1}^{\prime}$ of $G_{1}$, or alternatively $\gamma_{1}$ or $g_{1}$, as a function of the three other group elements. Also, in case the homomorphism $\iota_{1}$ is injective it alternatively fixes $h_{1} \in H_{1}$ in terms of the three other elements.,

We wish to obtain a 2-cocycle on the groupoid we have just described. For a general groupoid $\Gamma=\left(\Gamma_{0}, \Gamma_{1}\right)$ with sets $\Gamma_{0}$ of objects and $\Gamma_{1}$ of morphisms we define the 2-cocycle by its values $\tau\left(g_{1}, g_{1}^{\prime} ; \gamma\right)$ for an object $\gamma \in \Gamma_{0}$ and two compatible morphisms $g_{1}, g_{1}^{\prime} \in \Gamma_{1}$. We depict these values graphically as triangles,

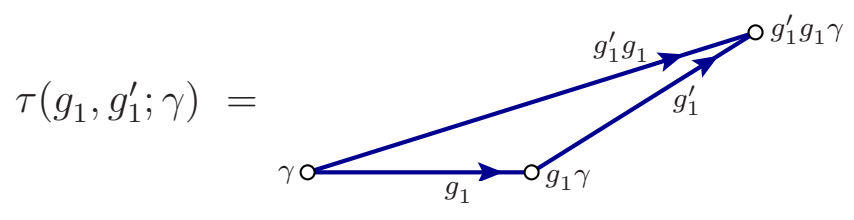

(Again the holonomy condition is in effect: we have $\left(g_{1}^{\prime} g_{1}\right)^{-1} g_{1}^{\prime} g_{1}=e$.)

Now in the situation of our interest, in which we represent objects and morphisms of the groupoid by one-dimensional and two-dimensional graphical elements, respectively, we obtain a graphical representation of the 2-cocycle by a piecewise-linear three-manifold. In the case of an interval considered in (3.28) - but now, for simplicity, with only a single interior marked point - this three-manifold looks as follows:

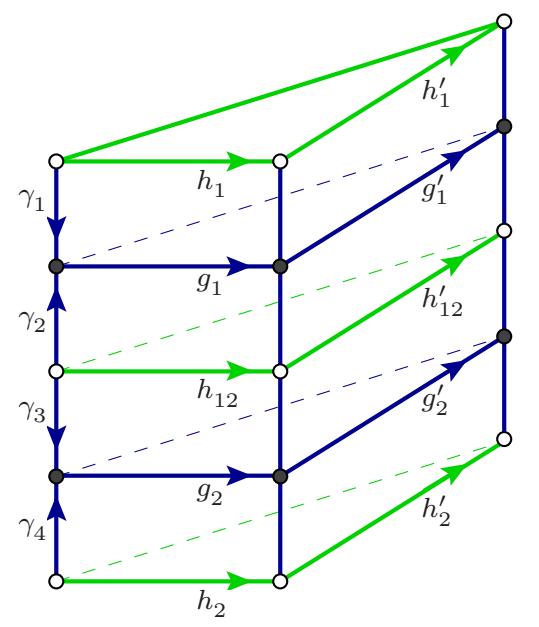

Here the labeling of all lines for which the labels are not indicated explicitly is fixed as a function of the displayed labels by the holonomy condition.

Following the strategy in [Wi], our goal is now to cut the so obtained three-manifolds into standard pieces to which we can naturally assign values in $\mathbb{C}^{\times}$. The value of the groupoid 2-cocycle is then given by the product of the numbers associated with the various standard pieces into which the three-manifold is decomposed. In our situation, in which also physical boundaries and surface defects are present, there are two types of standard pieces:

- First, a 3-simplex whose edges are all labeled by elements $g_{1}, g_{2}, g_{3}, \ldots$ of a group $G$ with 
3-cocycle $\omega \in Z^{3}\left(G, \mathbb{C}^{\times}\right)$, subject to the holonomy condition. To such a 3 -simplex

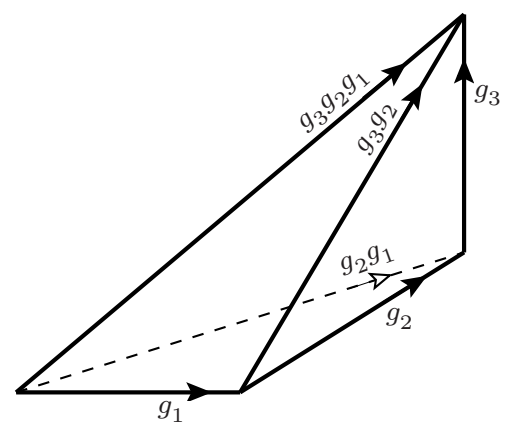

we associate the number

$$
\tilde{\omega}\left(g_{1}, g_{2}, g_{3}\right):=\omega\left(g_{1}^{-1}, g_{2}^{-1}, g_{3}^{-1}\right) \in \mathbb{C}^{\times} .
$$

- Second, a horizontal triangle whose edges are correspondingly labeled by elements of a group $H$ with 2-cochain $\theta$. To such a triangle

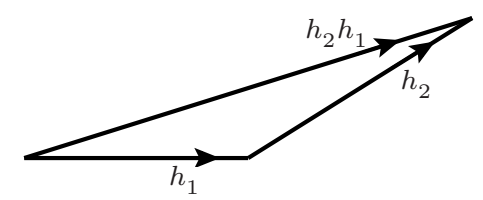

we associate the number

$$
\tilde{\theta}\left(h_{1}, h_{2}\right):=\left[\theta\left(h_{1}^{-1}, h_{2}^{-1}\right)\right]^{-1} \in \mathbb{C}^{\times} .
$$

We require that any horizontal triangle having only empty circles as vertices that is contained in a three-dimensional diagram of our interest must be taken as a face of the simplicial decomposition. The symmetric groups $S_{4}$ and $S_{3}$ which consist of permutations of the vertices in (3.33) and (3.35), respectively, are realized on $\tilde{\omega}$ and $\tilde{\theta}$ by a sign that depends on the relative orientations of the two bases involved, i.e. we have equalities such as

$$
\tilde{\omega}\left(g_{1}, g_{2}, g_{3}\right)=\tilde{\omega}\left(g_{1}^{-1} g_{2}^{-1} g_{3}^{-1}, g_{1}, g_{2}\right)^{-1}=\tilde{\omega}\left(g_{3}^{-1}, g_{2}^{-1}, g_{1}^{-1}\right)
$$

and

$$
\tilde{\theta}\left(h_{1}, h_{2}\right)=\tilde{\theta}\left(h_{1}^{-1} h_{2}^{-1}, h_{1}\right)=\tilde{\theta}\left(h_{2}^{-1}, h_{1}^{-1}\right)
$$

etc. We require that $\tilde{\omega}$ and $\tilde{\theta}$ are normalized, i.e.

$$
\tilde{\omega}\left(e, g, g^{\prime}\right)=1 \quad \text { and } \quad \tilde{\theta}(e, h)=1 .
$$

We will freely use the identities (3.37) - 3.39) below.

A simplicial decomposition obtained this way is not unique. We therefore must still verify that the value of the 2-cocycle on the groupoid that is obtained by our prescription is welldefined. When no boundaries or defects (and thus no triangular standard pieces) are involved, there are two situations to be dealt with: First, a gone with 5 vertices, 8 edges, 4 triangles and 
1 quadrangle. This gone can be decomposed into tetrahedra in two different ways; the first is a decomposition
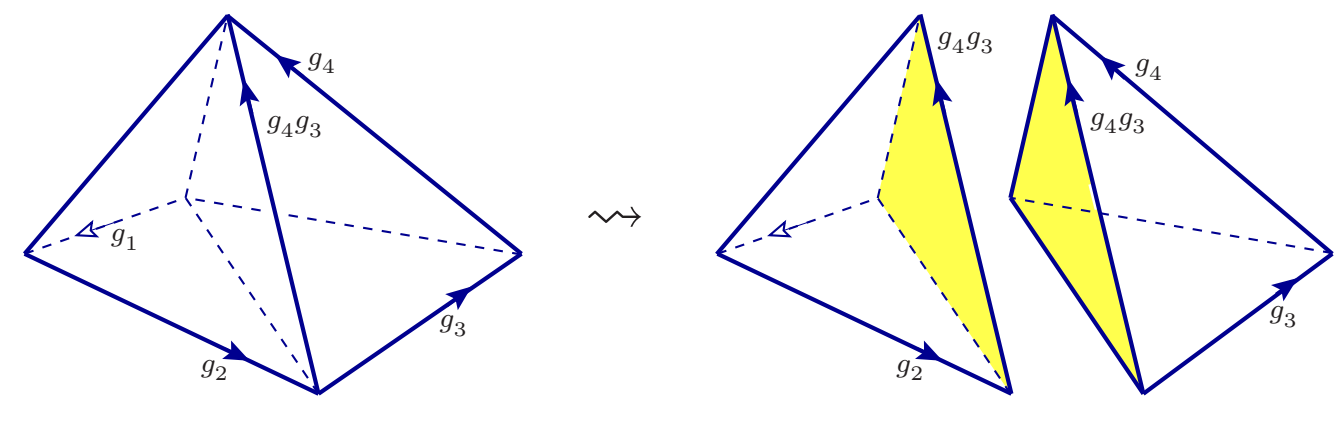

into two tetrahedra that share a face (shaded in the picture). The other is a decomposition is into three tetrahedra according to
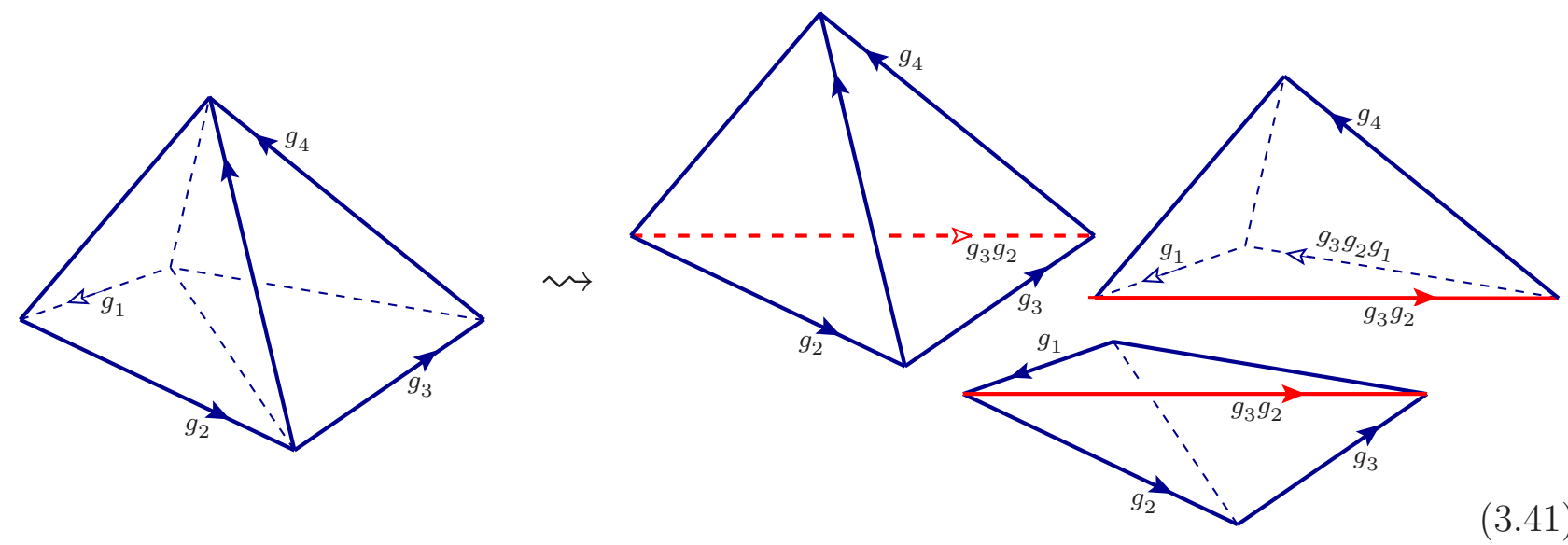

i.e. the three tetrahedra share an edge (the one labeled by $g_{3} g_{2}$ ) which intersects transversally the shaded face in (3.40) and pairwise share one of three faces which have the shared edge as a boundary segment.

The two decompositions are related by a 3-2 Pachner move. As is well known, invariance under this move is guaranteed by the closedness of $\omega$. Indeed we have

Lemma 3.4. The groupoid cocycles obtained from the two decompositions (3.40) and (3.41) coincide.

Proof. The decomposition (3.40) gives the number

$$
\tau_{1}:=\tilde{\omega}\left(g_{1}, g_{2}, g_{4} g_{3}\right) \cdot \tilde{\omega}\left(g_{2} g_{1}, g_{3}, g_{4}\right),
$$

while the decomposition (3.41) yields

$$
\tau_{2}:=\tilde{\omega}\left(g_{1}, g_{2}, g_{3}\right) \cdot \tilde{\omega}\left(g_{2}, g_{3}, g_{4}\right) \cdot \tilde{\omega}\left(g_{1}, g_{3} g_{2}, g_{4}\right),
$$

with the three factors being the contributions from the lower, the front, and the back tetrahedron, respectively. Equality of $\tau_{1}$ and $\tau_{2}$ amounts to

$$
\begin{aligned}
\omega\left(g_{1}^{-1}, g_{2}^{-1}, g_{3}^{-1} g_{4}^{-1}\right) & \cdot \omega\left(g_{1}^{-1} g_{2}^{-1}, g_{3}^{-1}, g_{4}^{-1}\right) \\
& =\omega\left(g_{1}^{-1}, g_{2}^{-1}, g_{3}^{-1}\right) \cdot \omega\left(g_{2}^{-1}, g_{3}^{-1}, g_{4}^{-1}\right) \cdot \omega\left(g_{1}^{-1}, g_{2}^{-1} g_{3}^{-1}, g_{4}^{-1}\right) .
\end{aligned}
$$

This is nothing but the statement that $\omega$ is closed, and is thus indeed satisfied. 
The second situation to be analyzed corresponds to a 4-1 Pachner move. It can be treated in an analogous manner as the 3-2 move; we leave the details to the reader.

Let us briefly comment on the particular case of the circle without insertions. According to Section 3.1, in this case the action groupoid is $G / /$ ad $G$ with the adjoint action. This situation is described by the simplex

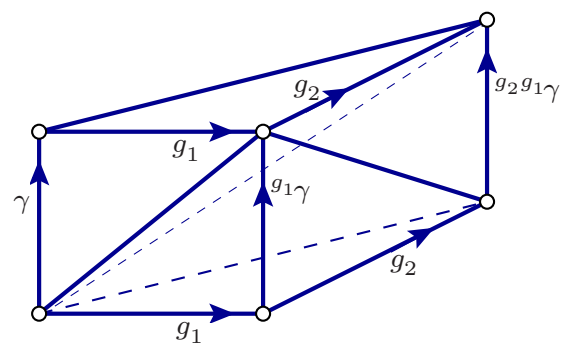

where we indicate the adjoint left action by a superscript, ${ }^{g} \gamma=g \gamma g^{-1}$. This yields the cocycle

$$
\tau\left(g_{1}, g_{2} ; \gamma\right)=\tilde{\omega}\left(g_{1}, g_{2},{ }_{2} g_{1} \gamma\right) \tilde{\omega}\left(g_{1},{ }^{g_{2}} \gamma, g_{2}\right)^{-1} \tilde{\omega}\left(\gamma, g_{1}, g_{2}\right) \text {. }
$$

This way we precisely recover the argument given in [Wi] that leads to the 2-cochain found in [DPR, (3.2.5)]. Our formalism thus produces the correct category of bulk Wilson lines.

We next consider the case of an interval with no marked interior points. The interior is labeled by a finite group $G$ and $\omega \in Z^{3}\left(G ; \mathbb{C}^{\times}\right)$, while the end points are labeled by group homomorphisms $\iota: H_{i} \rightarrow G$ and by 2-cochains $\theta_{i} \in C^{2}\left(H_{i}, \mathbb{C}^{\times}\right)$such that $\mathrm{d} \theta_{i}=\iota_{i}^{*} \omega$.

Again there is the issue of non-uniqueness of simplicial decomposition, with the new aspect that the boundary of the interval leads to the presence of triangles of the form (3.35) in the decompositions. Thus we must consider tetragons

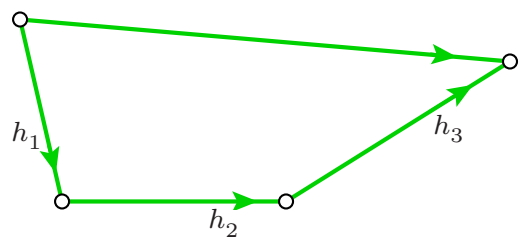

Such a boundary tetragon can be decomposed into triangles in two different ways: as

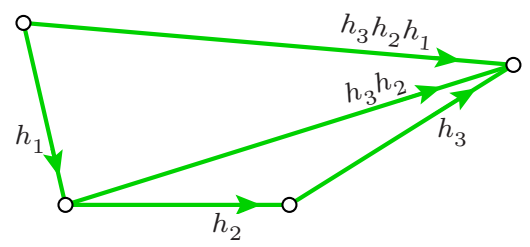

and as

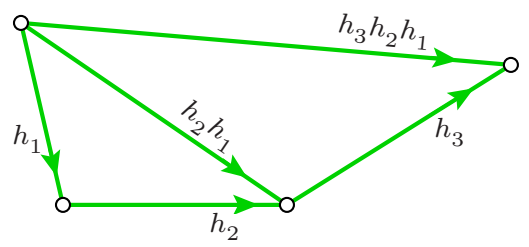

We compare these two decompositions by continuing the situation to the interior of the 
interval. This leads to the two simplicial decompositions
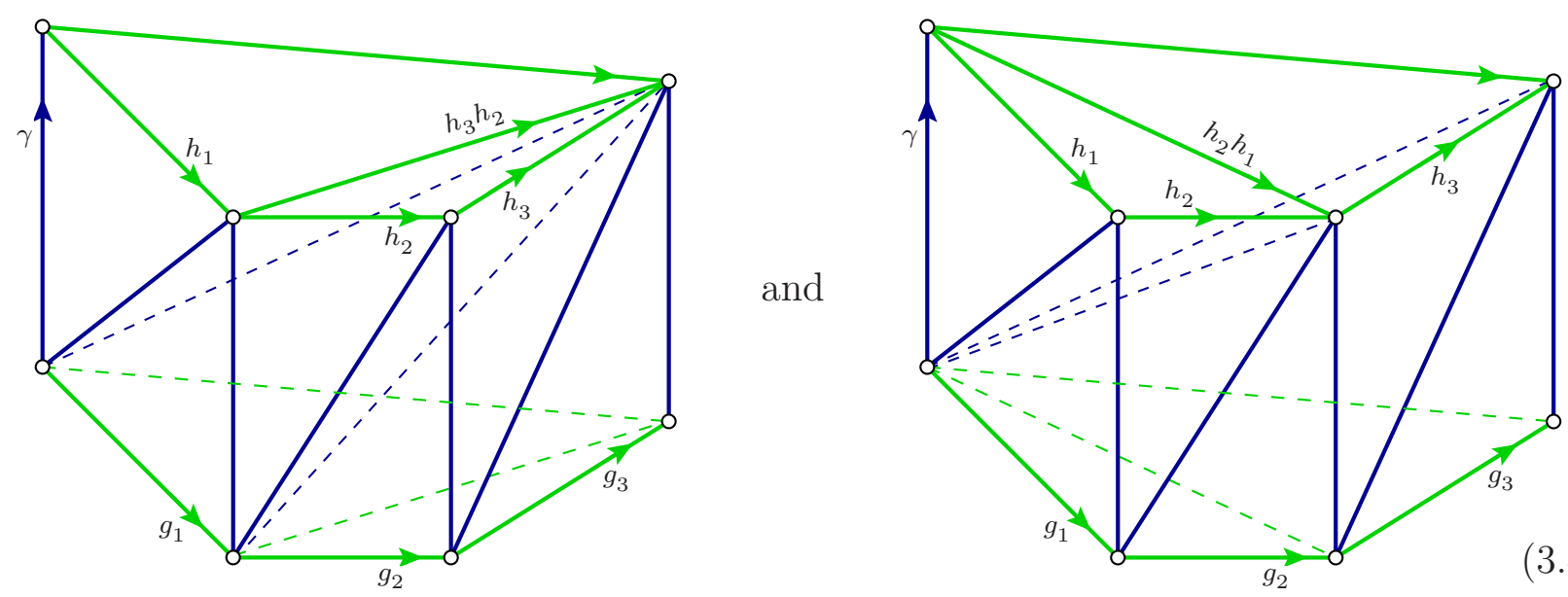

respectively, each consisting of six tetrahedra and of two triangles at the top.

Proposition 3.5. The complex numbers obtained from the two decompositions in (3.49) coincide.

Proof. Of the six tetrahedra appearing in the two simplices (3.49), only two are different: the ones attached to the top. The simplex on the right hand side of (3.49) gives factors $\tilde{\theta}\left(h_{1}, h_{2}\right)$ and $\tilde{\theta}\left(h_{2} h_{1}, h_{3}\right)$ from the triangles at the top and

$$
\tilde{\omega}\left(\gamma, \iota\left(h_{1}\right), \iota\left(h_{2}\right)\right) \cdot \tilde{\omega}\left(\gamma, \iota\left(h_{2} h_{1}\right), \iota\left(h_{3}\right)\right) .
$$

from the two tetrahedra attached to the top triangle, while for the simplex on the left hand side we get $\tilde{\theta}\left(h_{2}, h_{3}\right) \tilde{\theta}\left(h_{1}, h_{3} h_{2}\right)$ from the top triangles and

$$
\tilde{\omega}\left(\gamma, \iota\left(h_{1}\right), \iota\left(h_{3} h_{2}\right)\right) \cdot \tilde{\omega}\left(\iota\left(h_{1}\right) \gamma, \iota\left(h_{2}\right), \iota\left(h_{3}\right)\right)
$$

from the attached tetrahedra. Equality of the two expressions yields, after implementing the closedness (3.44) of $\omega$,

$$
\tilde{\theta}\left(h_{1}, h_{2}\right) \tilde{\theta}\left(h_{2} h_{1}, h_{3}\right)=\tilde{\theta}\left(h_{2}, h_{3}\right) \tilde{\theta}\left(h_{1}, h_{3} h_{2}\right) \tilde{\omega}\left(\iota\left(h_{1}\right), \iota\left(h_{2}\right), \iota\left(h_{3}\right)\right),
$$

or, what is the same

$$
\mathrm{d} \theta\left(h_{1}^{-1}, h_{2}^{-1}, h_{3}^{-1}\right)=\omega\left(\iota\left(h_{1}^{-1}\right), \iota\left(h_{2}^{-1}\right), \iota\left(h_{3}^{-1}\right)\right) .
$$

This indeed holds true, owing to $\mathrm{d} \theta=\iota^{*} \omega$.

\subsection{Wilson line categories for the interval}

As already pointed out, by invoking fusion of defects (and of defects to boundaries), among the one-dimensional manifolds there are two fundamental building blocks, the interval without interior marked points and the circle with a single marked point. We now turn to the computation of the categories for these building blocks and then compare them to the model-independent results of [FSV]. In the present subsection we consider an interval without interior marked 
points. The interior is labeled by $(G, \omega)$ with $G$ a finite group and $\omega$ a 3 -cocycle. For the two boundary points we have group homomorphisms $\iota_{i}: H_{i} \rightarrow G$ and 2-cochains $\theta_{i}$ on $H_{i}$ such that $\iota_{i}^{*} \omega=\mathrm{d} \theta_{i}$ for $i=1,2$.

Before computing the linearization, we outline what the general formalism of [FSV] predicts for the situation at hand: The data associated to a boundary leads to module categories $\mathcal{M}_{i}$ over the fusion category $G$-vect ${ }^{\omega}$. Such a module category can be decomposed into indecomposable module categories. As described in Section 2.5, an indecomposable module category over $G$-vect ${ }^{\omega}$ can, in turn, be concretely described Os1] as the category of modules over an algebra in $G$-vect ${ }^{\omega}$. Thus for the description of $\mathcal{M}_{i}$ it suffices to know such an algebra $A_{H, \theta}$ for any subgroup $H \leq G$ and 2-cochain $\theta$ on $H$ satisfying $\mathrm{d} \theta=\left.\omega\right|_{H}$. As seen in Section 2.5. such algebras can be described as follows. Isomorphism classes of simple objects in $G$-vect ${ }^{\omega}$ are in bijection with elements $g \in G$; we fix a set of representatives $\left(U_{g}\right)_{g \in G}$. Then $A_{H, \theta}$ is the object $\bigoplus_{h \in H} U_{h}$ endowed with the multiplication morphism that is furnished by the cochain $\theta$. This multiplication is associative, due to the relation $\mathrm{d} \theta=\iota^{*} \omega$. Then the category $\mathcal{M}_{H, \theta}:=A_{H, \theta^{-}} \bmod$ is a right module category over $G$-vect ${ }^{\omega}$.

By the results of [FSV], such a module category corresponds to an indecomposable boundary condition of the Dijkgraaf-Witten theory based on $(G, \omega)$. Given two such boundary conditions, consider the abelian category

$$
\mathcal{F}:=\operatorname{Fun}_{G-\operatorname{vect}} \omega\left(A_{H_{2}, \theta_{2}}-\bmod , A_{H_{1}, \theta_{1}}-\bmod \right)
$$

of module functors. It has the following physical interpretation: Objects of $\mathcal{F}$ label boundary Wilson lines separating the boundary condition $\mathcal{M}_{H_{1}, \theta_{1}}$ from $\mathcal{M}_{H_{2}, \theta_{2}}$. Morphisms of $\mathcal{F}$ label point-like insertions on such Wilson lines. $\mathcal{F}$ can be described as the category of $A_{H_{1}, \theta_{1}}-A_{H_{2}, \theta_{2}}{ }^{-}$ bimodules in $G$-vect ${ }^{\omega}$.

The objects $M=\bigoplus_{g \in G} M_{g}$ of the category of $A_{H_{1}, \theta_{1}}-A_{H_{2}, \theta_{2}}$-bimodules have been described explicitly in Os2, Prop.3.2]: Taking into account that the tensor product on $G$-vect ${ }^{\omega}$ realizes the group law strictly, i.e. $U_{h} \otimes U_{g}=U_{h g}$, the restriction of the left action of $A_{H_{1}, \theta_{1}}$ on $M$ to $U_{h_{1}} \otimes U_{g}$ leads to an endomorphism of $U_{h_{1} g}$ which is a multiple $\rho\left(h_{1}, g\right) \in \mathbb{C}$ of the identity. Analogously the right action of $A_{H_{2}, \theta_{2}}$ gives us scalars $q\left(g, h_{2}\right) \in \mathbb{C}$. These scalars obey the following conditions.

- That we have a left $A_{H_{1}, \theta_{1}}$-action amounts to the relation

$$
\rho\left(h_{1}^{\prime} h_{1}, g\right)=\theta_{1}\left(h_{1}^{\prime}, h_{1}\right)^{-1} \omega\left(h_{1}^{\prime}, h_{1}, g\right) \rho\left(h_{1}, g\right) \rho\left(h_{1}^{\prime}, h_{1} g\right)
$$

for all $g \in G$ and all $h_{1}, h_{1}^{\prime} \in H_{1}$.

- Similarly the right $A_{H_{2}, \theta_{2}}$-action gives

$$
\mathrm{q}\left(g, h_{2} h_{2}^{\prime}\right)=\theta_{2}\left(h_{2}, h_{2}^{\prime}\right)^{-1} \omega\left(g, h_{2}, h_{2}^{\prime}\right)^{-1} \mathrm{q}\left(g, h_{2}\right) \mathrm{q}\left(g h_{2}, h_{2}^{\prime}\right)
$$

for all $g \in G$ and all $h_{2}, h_{2}^{\prime} \in H_{2}$.

- The condition that left and right actions commute amounts to

$$
\rho\left(h_{1}, g\right) \mathrm{q}\left(h_{1} g, h_{2}\right)=\omega\left(h_{1}, g, h_{2}\right) \mathrm{q}\left(g, h_{2}\right) \rho\left(h_{1}, g h_{2}\right)
$$

for all $g \in G, h_{1} \in H_{1}$ and $h_{2} \in H_{2}$. 
- Finally the unitality of the actions implies the two constraints

$$
\rho(e, g)=1=\mathrm{q}(g, e)
$$

for all $g \in G$.

(Note that $\theta_{1}$ and $\theta_{2}$ are normalized because the algebras are strictly unital; (3.58) corresponds to $\omega$ being normalized as well.) The objects in the category $\mathcal{F}$ of $A_{H_{1}, \theta_{1}}-A_{H_{2}, \theta_{2}}$-bimodules are thus $G$-graded vector spaces together with two functions $\rho$ and $q$ that obey the constraints (3.55) - (3.58). Morphisms of $\mathcal{F}$ are $G$-homogeneous maps, commuting with the actions.

We may also consider, for given $\gamma \in G$, the group

$$
H_{\gamma}:=\left\{\left(h_{1}, h_{2}\right) \in H_{1} \times H_{2} \mid h_{1} \gamma=\gamma h_{2}\right\} .
$$

We can identify $H_{\gamma}$ with a subgroup of $H_{1}$, which in turn is a subgroup of $G$. Then $h \in H_{\gamma}$ acts on the homogeneous component $M_{\gamma}$ of $M$ as a scalar multiple

$$
\varrho_{\gamma}(h):=\rho(h, \gamma) q\left(\gamma, \gamma^{-1} h \gamma\right)^{-1}
$$

of the identity. In view of (3.55) - (3.57) this gives rise to a 2 -cocycle $\vartheta_{\gamma}$ on $H_{\gamma}$, given by

$$
\begin{aligned}
& \vartheta_{\gamma}\left(h, h^{\prime}\right):=\varrho_{\gamma}\left(h h^{\prime}\right)^{-1} \varrho_{\gamma}(h) \varrho_{\gamma}\left(h^{\prime}\right) \\
&=\rho\left(h h^{\prime}, \gamma\right)^{-1} \rho(h, \gamma) \rho\left(h^{\prime}, \gamma\right) q\left(\gamma, \gamma^{-1} h h^{\prime} \gamma\right) q\left(\gamma, \gamma^{-1} h \gamma\right)^{-1} q\left(\gamma, \gamma^{-1} h^{\prime} \gamma\right)^{-1} \\
&=\theta_{1}\left(h, h^{\prime}\right) \theta_{2}\left(\gamma^{-1} h \gamma, \gamma^{-1} h^{\prime} \gamma\right)^{-1} \omega\left(h, h^{\prime}, \gamma\right)^{-1} \omega^{-1}\left(\gamma, \gamma^{-1} h \gamma, \gamma^{-1} h^{\prime} \gamma\right) \\
& \rho(h, \gamma) \rho\left(h, h^{\prime} \gamma\right)^{-1} q\left(\gamma, \gamma^{-1} h^{\prime} \gamma\right)^{-1} q\left(h \gamma, \gamma^{-1} h^{\prime} \gamma\right) \\
&=\theta_{1}\left(h, h^{\prime}\right) \theta_{2}\left(\gamma^{-1} h^{\prime-1} \gamma, \gamma^{-1} h^{-1} \gamma\right) \\
& \omega\left(h, h^{\prime}, \gamma\right)^{-1} \omega\left(\gamma, \gamma^{-1} h \gamma, \gamma^{-1} h^{\prime} \gamma\right)^{-1} \omega\left(h, \gamma, \gamma^{-1} h^{\prime} \gamma\right)
\end{aligned}
$$

(compare formula (3.1) of [Os2]). Here in the third equality we invoke (3.55) and (3.56), while the last equality uses (3.57).

We now show that the prescription (3.2) indeed produces the expected result:

Proposition 3.6. Consider the groupoid $\Gamma=G \backslash G \times G / /_{\iota_{1}^{-} \times \iota_{2}^{-}} H_{1} \times H_{2}$ that according to formula (3.2) is assigned to the interval without interior marked points. If the group homomorphisms $\iota_{i}: H_{i} \rightarrow G$ are subgroup embeddings, then the category that is obtained by the projective linearization of $\Gamma$ for the Lagrangian data $\theta_{1}, \theta_{2}$ and $\omega$ is equivalent, as a $\mathbb{C}$-linear abelian category, to the category of $A_{H_{1}, \theta_{1}}-A_{H_{2}, \theta_{2}}$-bimodules,

$$
\left[G \backslash G \times G / /_{\iota_{1}^{-} \times \iota_{2}^{-}} H_{1} \times H_{2}, \text { vect }_{\mathbb{C}}\right]^{\theta_{1}, \theta_{2}, \omega} \simeq A_{H_{1}, \theta_{1}}-A_{H_{2}, \theta_{2}}-\operatorname{Bimod}_{G \text {-vect }}{ }^{\omega} .
$$

Proof. The objects of the groupoid in question are pairs $\left(\gamma_{1}, \gamma_{2}\right)$ of elements of $G$; they label 
the vertical edges in the following figure:

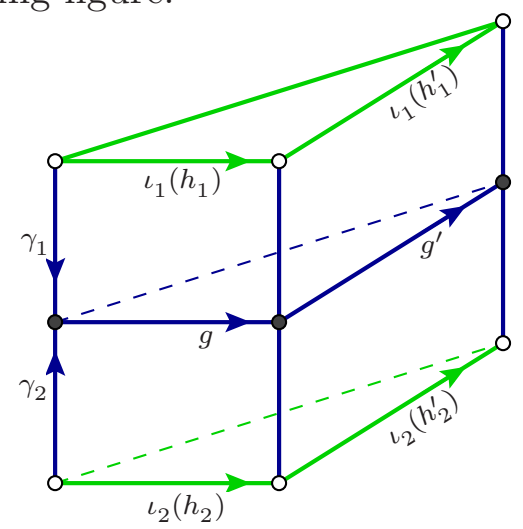

Morphisms are gauge transformations in $H_{1}, H_{2}$ and in $G$ - labeling horizontal edges that connect empty circles and filled circles in (3.63), respectively. Again we consider a pair of compatible morphisms leading to horizontal edges forming the shape of a triangle to get the relevant 2 -cocycle on the groupoid $\Gamma$. In the sequel we suppress the embedding homomorphisms $\iota_{1}$ and $\iota_{2}$.

Observe that the functor

$$
G \backslash \backslash G \times G / \iota_{\iota_{1}^{-} \times \iota_{2}^{-}} H_{1} \times H_{2} \longrightarrow H_{1} \iota_{1} \backslash \backslash G / \iota_{2}^{-} H_{2}
$$

that is defined on objects by $\left(\gamma_{1}, \gamma_{2}\right) \mapsto \gamma_{1}^{-1} \gamma_{2}$ is actually an equivalence of groupoids. Accordingly we set $\gamma:=\gamma_{1}^{-1} \gamma_{2}$ and obtain from (3.63) a number $\tau\left(\gamma ; h_{1}, h_{1}^{\prime} ; h_{2}, h_{2}^{\prime}\right)$ that can be read off from the following slice of pie:

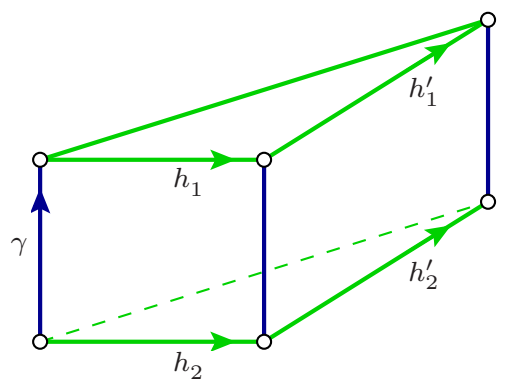

where $\gamma \in G, h_{1}, h_{1} \in H_{1}$ and $h_{2}, h_{2}^{\prime} \in H_{2}$. There are many equivalent ways to express the so defined numbers in terms of the 2-cocycles $\theta_{i}$ and the 3-cocycle $\omega$; they are related by the various properties of $\theta_{i}$ and $\omega$. Let us choose one such expression that corresponds to the decomposition

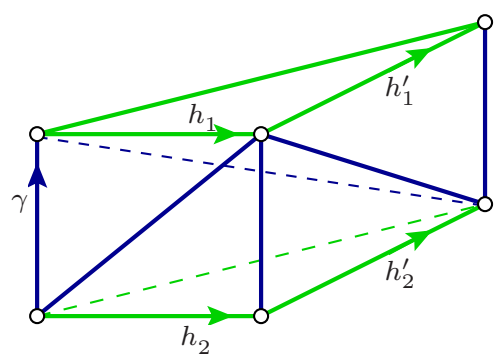

of the slice (3.65) into three tetrahedra. This yields

$$
\begin{aligned}
\tau\left(\gamma ; h_{1}, h_{1}^{\prime} ; h_{2}, h_{2}^{\prime}\right)=\tilde{\theta}_{1}\left(h_{1}, h_{1}^{\prime}\right) \tilde{\theta}_{2}\left(h_{2}, h_{2}^{\prime}\right) \tilde{\omega}\left(h_{1}, h_{1}^{\prime}, h_{2}^{\prime} h_{2} \gamma^{-1} h_{1}^{-1} h_{1}^{\prime-1}\right) \\
\tilde{\omega}\left(h_{2}, h_{2}^{\prime}, h_{1} \gamma h_{2}^{-1} h_{2}^{\prime-1}\right) \tilde{\omega}\left(\gamma, h_{1}, h_{2}^{\prime} h_{2} \gamma^{-1} h_{1}^{-1}\right) .
\end{aligned}
$$


To make contact to the relations (3.55) - (3.57) for the category of $A_{H_{1}, \theta_{1}}-A_{H_{2}, \theta_{2}}$-bimodules, we consider three special cases of $\tau\left(\gamma ; h_{1}, h_{1}^{\prime} ; h_{2}, h_{2}^{\prime}\right)$.

- First we set $h_{2}=e=h_{2}^{\prime}$; then (3.67) reduces to

$$
\begin{aligned}
\tau\left(\gamma ; h_{1}, h_{1}^{\prime} ; e, e\right) & =\tilde{\theta}_{1}\left(h_{1}^{\prime-1}, h_{1}^{-1}\right) \tilde{\omega}\left(h_{1}, h_{1}^{\prime}, \gamma^{-1} h_{1}^{-1} h_{1}^{\prime-1}\right) \tilde{\omega}\left(\gamma, h_{1}, \gamma^{-1} h_{1}^{-1}\right) \\
& =\tilde{\theta}_{1}\left(h_{1}^{\prime-1}, h_{1}^{-1}\right) \tilde{\omega}\left(h_{1}^{\prime-1}, h_{1}^{-1}, \gamma^{-1}\right)=\theta_{1}\left(h_{1}^{\prime}, h_{1}\right)^{-1} \omega\left(h_{1}^{\prime}, h_{1}, \gamma\right)
\end{aligned}
$$

This reproduces the factor in the relation (3.55) for the left action of $H_{1}$, with $g=\gamma$.

- Next consider the case $h_{1}=e=h_{1}^{\prime}$; then we get

$$
\begin{aligned}
\tau\left(\gamma ; e, e ; h_{2}, h_{2}^{\prime}\right) & =\tilde{\theta}_{2}\left(h_{2}, h_{2}^{\prime}\right) \tilde{\omega}\left(h_{2}, h_{2}^{\prime}, \gamma h_{2}^{-1} h_{2}^{\prime-1}\right) \\
& =\tilde{\theta}_{2}\left(h_{2}, h_{2}^{\prime}\right) \tilde{\omega}\left(\gamma^{-1}, h_{2}, h_{2}^{\prime}\right)^{-1}=\theta_{2}\left(h_{2}^{-1}, h_{2}^{\prime-1}\right)^{-1} \omega\left(\gamma, h_{2}^{-1}, h_{2}^{\prime-1}\right)^{-1} .
\end{aligned}
$$

This is the factor in (3.56), provided we replace the group elements $h_{2}$ and $h_{2}^{\prime}$ in (3.56) by their inverses, which is precisely what is needed to turn the right action of $H_{2}$ in (3.56) to the left action considered here.

- Finally take $h_{1}^{\prime}=e=h_{2}^{\prime}$. This results in

$$
\tau\left(\gamma ; h_{1}, e, h_{2}, e\right)=\tilde{\omega}\left(\gamma, h_{1}, h_{2} \gamma^{-1} h_{1}^{-1}\right)=\tilde{\omega}\left(h_{1}^{-1}, \gamma^{-1}, h_{2}\right)=\omega\left(h_{1}, \gamma, h_{2}^{-1}\right),
$$

thus reproducing the factor appearing in the bimodule relation (3.57) (again upon putting $g=\gamma$ and inverting $h_{2}$ ).

Notice that the number $\tilde{\omega}\left(h_{1}^{\prime-1}, h_{1}^{-1}, \gamma^{-1}\right)$ appearing in the expression (3.68) corresponds to a tetrahedron that can be viewed as the degeneration of the slice (3.65) that results from the degeneration of its bottom triangle to a single point. Similarly, $\tilde{\omega}\left(\gamma^{-1}, h_{2}, h_{2}^{\prime}\right)^{-1}$ in (3.69) corresponds to the degeneration of the top triangle of (3.65) to a point. And the tetrahedron corresponding to $\tilde{\omega}\left(h_{1}^{-1}, \gamma^{-1}, h_{2}\right)$ in (3.70) can be obtained by gluing together two quadrangles along their edges which are obtained from the slice (3.65) by degenerating both the top and the bottom triangle to a single edge.

\subsection{The transparent defect}

We now address aspects of categories associated to DW manifolds with the topology of a circle. Recall that one expects that surface defects can be fused and should thus form a monoidal bicategory. We refer to the monoidal unit of this monoidal bicategory as the transparent, or invisible surface defect. We have already mentioned in Section 2.2 that in the framework of [FSV] the transparent surface defect should correspond to the canonical Witt trivialization (2.17). In the present subsection we are interested in the Lagrangian realization of this distinguished surface defect.

To understand what group homomorphism and 2-cocycle furnish the transparent defect, we consider a circle with any number $n$ of surface defects, one of which is transparent. By fusing all other surface defects to a single one, we can reduce the situation to the case $n=2$. This situation has already been studied in Section 3.1; it leads to the groupoid (3.7). To realize the transparent defect for one of the two marked points we must moreover set $G_{>}=G_{<}=$: $G$ and 
take the same 3-cocycle $\omega$ on either side. Now we claim that the group homomorphism for the transparent defect is the diagonal subgroup embedding, i.e. we have to set $H_{+}=G$ with $\iota_{+}=d: G \rightarrow G \times G$ the diagonal embedding. This way we arrive at the action groupoid

$$
\Gamma_{1}:=G \times G \backslash \backslash G \times G \times G \times G / / d^{-} \times \iota^{-} G \times H
$$

which we already considered in (3.8). We further claim that the relevant 2-cochain on $H=G$ is the constant 2-cochain $\theta_{d} \equiv 1$. Note that this is a valid cochain, as it satisfies $\mathrm{d} \theta_{d}=1=\omega \cdot \omega^{-1}$.

To see that the defect defined by $\iota=d$ and $\theta=1$ indeed has the relevant properties of the transparent defect, recall first that in (3.10) we have obtained an equivalence $F: \Gamma_{1} \stackrel{\simeq}{\rightarrow} \Gamma_{2}$ between $\Gamma_{1}$ and the action groupoid

$$
\Gamma_{2}:=G \backslash \backslash G \times G / \iota_{\llcorner} H
$$

introduced in (3.9), and that the latter groupoid is precisely the one relevant for the circle with a single surface defect of arbitrary type. Our prescription also yields 2-cocycles $\tau_{1}$ on $\Gamma_{1}$ and $\tau_{2}$ on $\Gamma_{2}$. We need to show that we still get an equivalence after linearization with respect to Lagrangian data. To this end, describe the second defect by $(H, \theta)$ with group homomorphisms $\iota_{i}: H \rightarrow G$ and a 2 -cochain $\theta$ on $H$ satisfying $\mathrm{d} \theta=\left(\iota_{1}^{*} \omega\right)\left(\iota_{2}^{*} \omega\right)^{-1}$. We then have

Proposition 3.7. The pullback along the functor $F: \Gamma_{1} \rightarrow \Gamma_{2}$ described in (3.10) yields an equivalence

$$
\begin{aligned}
F^{*}: \quad\left[\Gamma_{2}, \text { vect }\right]^{\tau_{2}} & \stackrel{\simeq}{\longrightarrow}\left[\Gamma_{1}, \text { vect }\right]^{\tau_{1}} \\
\varphi & \longmapsto \varphi \circ F
\end{aligned}
$$

of $\mathbb{C}$-linear abelian categories.

Proof. Morphisms in the groupoid $\Gamma_{1}$ have the form (3.11). Pick two such morphisms $\left(g_{1}, g_{2}, g, h\right)$ and $\left(g_{1}^{\prime}, g_{2}^{\prime}, g^{\prime}, h^{\prime}\right)$. Their images under $F$ are morphisms $\left(g_{1}, h\right)$ and $\left(g_{1}^{\prime}, h^{\prime}\right)$ in $\Gamma_{2}$, of the form (3.12). We must show that

$$
\tau_{1}\left(\gamma_{1}, \gamma_{2}, \gamma_{3}, \gamma_{4} ; g_{1}, g_{2}, g, h ; g_{1}^{\prime}, g_{2}^{\prime}, g^{\prime}, h^{\prime}\right)=\tau_{2}\left(\gamma_{1} \gamma_{2}^{-1} \gamma_{3}, \gamma_{4} ; g_{1}, h ; g_{1}^{\prime}, h^{\prime}\right)
$$

for all quadruples $\left(\gamma_{1}, \gamma_{2}, \gamma_{3}, \gamma_{4}\right)$ of elements of $G$. Both sides of (3.74) are obtained by evaluating appropriate diagrams of the form of slices of pie with top and bottom faces identified. The diagram relevant to $\Gamma_{1}$ is similar to the one of figure (3.32), but now with identified top and bottom, so that $h_{1}=h_{2}=: h$ and $h_{1}^{\prime}=h_{2}^{\prime}=: h^{\prime}$, as well as with $h_{12}=g$ and $h_{12}^{\prime}=g^{\prime}$ being now elements of $G$; this diagram is shown on the left hand side of the picture (3.75) below. In the case of $\Gamma_{2}$ there is, besides the identified top and bottom faces, only one horizontal face, with edges labeled by elements $g_{1}$ and $g_{1}^{\prime}$ of $G$; this diagram is shown on the right hand side of the 
picture:
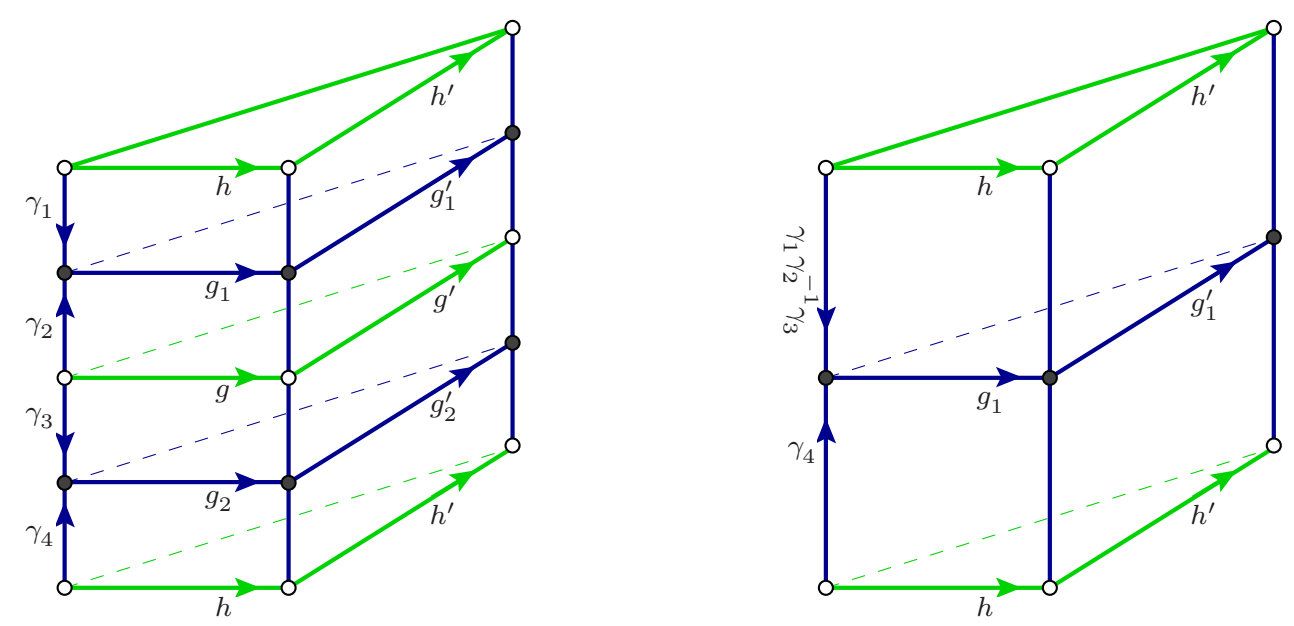

It should be appreciated that the two diagrams only differ in a part that is of the same topology and only involves edges labeled by $G$. It is easily seen that there is a sequence of Pachner moves relating the decompositions of the two diagrams in (3.75). And as discussed in Section 3.4, invariance under Pachner moves holds (as a direct consequence of the axioms of group cohomology) for the decomposition of simplices into tetrahedra. Together it follows that indeed the 2-cocycles on the left and right hand sides of (3.74) have the same value.

To summarize our findings: The surface defect labeled by $\iota=d$ and $\theta=1$ can be omitted without changing the category that our linearization procedure associates to the circle. In other words, it has the characteristic property of the monoidal unit for the fusion of surface defects, and thus of the transparent defect.

\subsection{Wilson line categories for the circle}

A one-dimensional DW manifold with the topology of a circle can contain finitely many marked points, corresponding to surface defects. Invoking fusion of defects, the situation with any number of marked points can be reduced to the one with a single marked point, which thereby constitutes one of the two fundamental building blocks. In this subsection we finally compute the category of generalized Wilson lines corresponding to this building block and compare it with the results of [FSV] for defects in topological field theories of Reshetikhin-Turaev type.

Let, as before, the subinterval be labeled by $(G, \omega)$ and the defect by a group homomorphism $\iota: H \rightarrow G \times G$ and a 2 -cochain $\theta$ on $H$ satisfying $\mathrm{d} \theta=\left(\iota_{1}^{*} \omega\right)\left(\iota_{2}^{*} \omega\right)^{-1}$. We can restrict our attention to indecomposable defects and therefore assume that $\iota$ is injective. For this situation our formalism yields in a straightforward manner the groupoid

$$
G \backslash \backslash G \times G / /^{-} H
$$

that we already encountered in (3.9). Its (projective) linearization, which we denote by $\mathcal{W}_{H, \theta}$, is the abelian category of $G \times G$-graded vector spaces with two commuting left actions (which are, in general, projective): a left action of $G$ such that $g . V_{\gamma_{1} \gamma_{2}} \subseteq V_{g \gamma_{1}, g \gamma_{2}}$ and a left $H$-action such that $h . V_{\gamma_{1} \gamma_{2}} \subseteq V_{\gamma_{1} \iota_{1}(h)^{-1}, g \gamma_{2} \iota_{2}(h)^{-1}}$.

The category $\mathcal{W}_{H, \theta}$ has the interpretation of the category of generalized Wilson lines separating the defect labeled by $\iota$ and $\theta$ from the transparent defect that we studied in the previous 
subsection. Pictorially, fusion of surface defects replaces the configuration depicted on the right hand side of figure (3.1), in which four surface defects meet in a generalized Wilson line, by the configuration shown in the following picture, in which the single non-trivial defect is on the right and the transparent defect on the left:

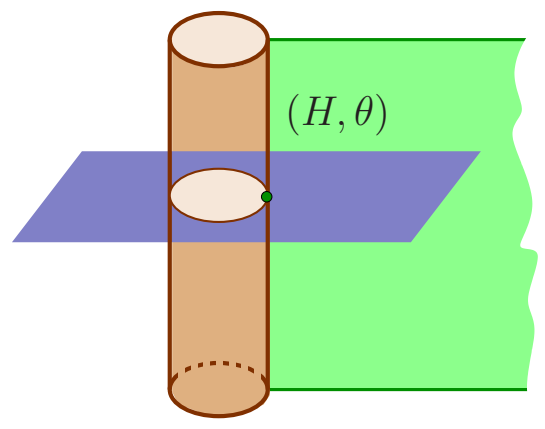

We claim that the category produced by our geometric prescription is the same as the Wilson line category that is obtained in the formalism of [FSV]. Let us thus compute the latter. According to formula (2.12), in the framework of [FSV] a surface defect is described by a Witt trivialization. Now in the case of Dijkgraaf-Witten theories already the modular tensor category $\mathcal{C}$ of bulk Wilson lines is, by definition, Witt trivial. Indeed, $\mathcal{C}=\mathcal{Z}(\mathcal{A})$, where for the theory based on $(G, \omega), \mathcal{A}$ is the fusion category of finite-dimensional $G$-graded vector spaces with associativity constraint given by $\omega$ as in (2.47). It is not difficult to verify that the Witt trivialization of $\mathcal{C}$ implies the Witt trivialization

$$
\mathcal{C} \otimes \mathcal{C}^{\text {rev }} \stackrel{\simeq}{\longrightarrow}\left(\mathcal{A} \otimes \mathcal{A}^{\text {op }}\right)
$$

where $\mathcal{A}^{\text {op }}$ is the fusion category $\mathcal{A}$ with opposite tensor product.

Indecomposable surface defects separating the modular tensor category $\mathcal{C}=\mathcal{Z}(\mathcal{A})$ from itself correspond [FSV] to indecomposable module categories over $\mathcal{A} \otimes \mathcal{A}^{\text {op }}$ which is, as an abelian category, the category of $G \times G$-graded vector spaces. According to the results reported in Section 2.5, such a module category is described by a subgroup $H \leq G \times G$ and a 2-cochain $\theta$ on $H$. This category can be realized as $\mathcal{M}_{H, \theta}=A_{H, \theta}$-mod, with the algebra $A_{H, \theta}$ as introduced in Section 2.5. The category $\mathcal{M}_{H, \theta}$ of $A_{H, \theta}$-modules, seen as a module category over $\mathcal{A} \otimes \mathcal{A}^{\mathrm{op}}$, describes the non-transparent surface defect in the situation we are considering.

The analogous algebra in $\mathcal{A} \otimes \mathcal{A}^{\text {op }}$ that is relevant for the transparent defect can be deduced from the discussion in Section 3.6, it is the algebra $A_{d}$ for the diagonal subgroup $G \leq G \times G$ with trivial 2-cocycle $\theta=1$. The category of Wilson lines described by the linearization of the groupoid (3.76) should therefore be matched to the category

$$
\operatorname{Hom}_{\mathcal{A} \otimes \mathcal{A}^{\text {op }}}\left(\mathcal{A}, \mathcal{M}_{H, \theta}\right)
$$

of module functors or, equivalently, to the category of $A_{d^{-}} A_{H, \theta^{-}}$bimodules in $\mathcal{A} \otimes \mathcal{A}^{\mathrm{op}}$. But the latter is nothing else than the category of $G \times G$-graded vector spaces together with projective actions of $H$ and $G$.

This concludes the match of the categories that are obtained, for the case of the circle, in the present geometric approach and in [FSV]. 


\section{A Module categories for non-injective group homomor- phisms}

As described in Section 2.5, indecomposable module categories over the fusion category $G$-vect are given by subgroups $H \leq G$ and group cochains. On the other hand, in the definition of relative bundles a group homomorphism $\iota: H \rightarrow G$ enters. In the geometric context, it is not natural, and for many purposes, e.g. for the discussion of fusion of surface defects, not appropriate, to require $\iota$ to be injective. This raises the question how corresponding module categories decompose into indecomposable ones if the group homomorphism $\iota$ is not injective. We discuss this issue in the simplest setting, in particular dropping Lagrangian data.

We consider a morphism $\iota: H \rightarrow G$ of finite groups and the action groupoid $G / \iota^{-} H$ with left action $h \cdot \gamma=\gamma \iota(h)^{-1}$. The functor category $\mathcal{M}:=\left[G / l_{\iota} H\right.$, vect $]$ is a module category over the monoidal category $G$-vect as follows. Objects in $\mathcal{M}$ are $G$-graded vector spaces $V=\bigoplus_{g \in G} V_{g}$ endowed with a left action of $H$ such that

$$
h . V_{g} \subset V_{g \cdot \iota(h)^{-1}} .
$$

The simple object $W_{\gamma}$ of $G$-vect acts on such an object of $\mathcal{M}$ by shifting the degrees of the homogeneous components by left multiplication by $\gamma$ and keeping the action of $H$ :

$$
\left(W_{\gamma} \otimes V\right)_{g}=V_{\gamma \cdot g}
$$

Any module category over $G$-vect can be decomposed into indecomposable module categories. Let us see how this works for the module categories arising in the way considered here. To this end we consider the normal subgroup $K:=\operatorname{ker} \iota \leq H$ and the exact sequence

$$
1 \rightarrow K \rightarrow H \stackrel{\pi}{\rightarrow} J \rightarrow 1
$$

of groups. This sequence is, in general, not split, and $H$ is thus not a semidirect product. Still, we can choose a set-theoretic section $s: J \rightarrow H$ of $\pi$, which for convenience we require to respect neutral elements, $s\left(e_{J}\right)=e_{H}$. We keep the section $s$ fixed from now on. For each $j \in J$ consider the group automorphism

$$
\alpha_{j}:=\left.\operatorname{ad}_{s(j)}\right|_{K} \in \operatorname{Aut}(K) .
$$

The automorphism $\alpha_{j}$ is not necessarily inner; its class $\left[\bar{\alpha}_{j}\right] \in \operatorname{Out}(K)=\operatorname{Aut}(K) / \operatorname{Inn}(K)$ does not depend on the choice of $s$. Moreover, introduce group elements

$$
c_{i, j}:=s(i) s(j) s(i j)^{-1} \in K
$$

for each pair $i, j \in J$. Then one has the relation

$$
\alpha_{j} \circ \alpha_{j^{\prime}}=\operatorname{ad}_{c_{j, j^{\prime}}} \circ \alpha_{j j^{\prime}}
$$

and obvious coherence conditions on the elements $c_{i j} \in K$; thus $\left(\alpha_{j}, c_{i, j}\right)$ defines a weak action of the group $J$ on the group $K$. We use this observation to rewrite the group $H$ in a convenient way. The map

$$
\begin{aligned}
\psi: \quad J \times K & \rightarrow H \\
(j, k) & \mapsto k \cdot s(j)
\end{aligned}
$$


has the inverse

$$
\begin{aligned}
\psi^{-1}: \quad H & \rightarrow J \times K \\
h & \mapsto\left(\pi(h), h \cdot(s \pi(h))^{-1}\right) .
\end{aligned}
$$

Define on the set $J \times K$ a composition map

$$
(i, k) \cdot\left(j, k^{\prime}\right):=\left(i j, k \alpha_{i}\left(k^{\prime}\right) c_{i j}\right) .
$$

A direct calculation shows that the map $\psi$ is compatible with the product (A.9) and with the product on $H$. Thus (A.9) endows the set $J \times K$ with the structure of a finite group isomorphic to $H$. We denote this group structure by $J \times_{\alpha} K$, suppressing the group elements $c$ in the notation. We will identify $J \cong G / K$ with a subgroup of $G$ in the sequel.

Thus we now replace $H$ by the isomorphic group $J \times_{\alpha} K$. Then the left $J \times_{\alpha} K$-action on $V=\bigoplus_{g \in G} V_{g}$ satisfies

$$
(j, k)\left(V_{g}\right) \subset V_{g \cdot j^{-1}}
$$

Moreover, each homogeneous component $V_{g}$ has a natural structure of a $K$-module from the action of elements of the form $\left(e_{J}, k\right) \in J \times_{\alpha} K$.

It is crucial to note that the so obtained $K$-module structures on different homogeneous components $V_{g}$ are in general not isomorphic. They are related by the action of elements of the form $(j, k)$ that are twisted intertwiners rather than morphisms of $K$-modules. Comparing the group elements $(e, k) \cdot\left(j, k^{\prime}\right)=\left(j, k k^{\prime}\right)$ and $\left(j, k^{\prime}\right)\left(e, k^{\prime \prime}\right)=\left(j, k^{\prime} \alpha_{j}\left(k^{\prime \prime}\right)\right)$ we deduce that

$$
(e, k) \cdot\left(j, k^{\prime}\right)=\left(j, k^{\prime}\right)\left(e, k^{\prime \prime}\right) \quad \text { with } \quad k^{\prime \prime}=\alpha_{j}^{-1}\left(\left(k^{\prime}\right)^{-1} k k^{\prime}\right) .
$$

Thus the action by $\left(j, k^{\prime}\right)$ is a twisted intertwiner relating a $K$-module in the isomorphism class $\left[V_{g}\right]$ to a $K$-module in the class $\left[V_{g . j}\right]=\bar{\alpha}_{j}^{-1}\left[V_{g}\right]$. These two isomorphism class are different if $\alpha_{j}$ is outer.

To find the simple objects of the category $\left[G / /_{\iota^{-}} H\right.$, vect], fix representatives $\left(\gamma_{1}, \gamma_{2}, \ldots, \gamma_{r}\right)$ for the orbits of the right action of $J$ on $G$. Then the isomorphism classes of simple objects are in bijection with pairs $\left(\gamma_{i}, \chi\right)$ with $\chi \in \widehat{K}$ a simple character of $K$. The action of $G$-vect on the set of isomorphism classes of simple objects $\left(\gamma_{i}, \chi\right)$ of the category $\left[G / l_{-}-H\right.$, vect] and thus its decomposition as a module category over $G$-vect can now be computed explicitly.

An instructive example is the group homomorphism $\iota: H=S_{3} \rightarrow \mathbb{Z}_{2}=G$, with $S_{3}$ the symmetric group on three letters, that is given by the sign function. The exact sequence (A.3) of groups is then

$$
1 \longrightarrow A_{3} \cong \mathbb{Z}_{3} \longrightarrow S_{3} \stackrel{\text { sign }}{\longrightarrow} \mathbb{Z}_{2} \longrightarrow 1
$$

The simple objects of the resulting linearization $\left[\mathbb{Z}_{2} / / S_{3}\right.$, vect $]$ are labeled by the single orbit of the right action of $\mathbb{Z}_{2}$ on itself and by one of the three irreducible characters $\left\{1, \zeta, \zeta^{\vee}\right\}$ of $\mathbb{Z}_{3}$. Since $S_{3}$ is a semidirect product, any section $s: \mathbb{Z}_{2} \rightarrow S_{3}$, e.g. the one mapping the generator of $\mathbb{Z}_{2}$ to the permutation $\tau_{12} \in S_{3}$, gives a genuine action of $\mathbb{Z}_{2}$ on $\mathbb{Z}_{3}$, rather than only a weak action. Here the generator of $\mathbb{Z}_{2}$ acts as the outer automorphism of $\mathbb{Z}_{3}$ which exchanges the nontrivial irreducible characters $\zeta$ and $\zeta^{\vee}$. This fixes the $\mathbb{Z}_{3}$-representation on the homogeneous component $V_{1}$ in terms of the $\mathbb{Z}_{2}$-representation on $V_{0}$ as shown in the following table:

\begin{tabular}{cc} 
rep. on $V_{0}$ & rep. on $V_{1}$ \\
\hline 1 & 1 \\
$\zeta$ & $\zeta^{\vee}$ \\
$\zeta^{\vee}$ & $\zeta$
\end{tabular}


We conclude that the abelian category $\left[\mathbb{Z}_{2} / / S_{3}\right.$, vect] has three isomorphism classes of simple objects, corresponding to the three lines of the table.

To determine the structure of $\left[\mathbb{Z}_{2} / / S_{3}\right.$, vect $]$ as a module category over $\mathbb{Z}_{2}$-vect we note that the action of the simple object $X_{g}$ in a non-trivial homogeneous component exchanges the two homogeneous components $V_{0}$ and $V_{1}$. It therefore preserves the isomorphism class of simple $\left[\mathbb{Z}_{2} / / S_{3}\right.$, vect $]$-objects in the first line of $(\underline{A} .13)$ and exchanges the two classes in the other two lines. Thus the first line of (A.13) gives us one indecomposable module category over $\mathbb{Z}_{2}$-vect with a single simple object, which corresponds to $\mathbb{Z}_{2}$ seen as a subgroup of itself. From the second and third lines of (A.13) we get another indecomposable module category having two simple objects, corresponding to the trivial subgroup $\{e\}$ of $\mathbb{Z}_{2}$.

Acknowledgments: We thank Domenico Fiorenza, Jeffrey Morton and Jan Priel for helpful discussions. JF is still to some extent supported by VR under project no. 621-2009-3993. CS and AV are partially supported by the Collaborative Research Centre 676 "Particles, Strings and the Early Universe - the Structure of Matter and Space-Time" and by the DFG Priority Programme 1388 "Representation Theory". JF is grateful to Hamburg University, and in particular to CS, Astrid Dörhöfer and Eva Kuhlmann, for their hospitality when part of this work was done. 


\section{References}

[BJQ] M. Barkeshli, C.M. Jian, and X.L. Qi, Theory of defects in Abelian topological states, Phys. Rev. B 88 (2013) 235103-1-21 [cond-mat/1305.7203]

[DPR] R. Dijkgraaf, V. Pasquier, and P. Roche, Quasi Hopf algebras, group cohomology and orbifold models, Nucl. Phys. B (Proc. Suppl.) 18B (1990) 60-72

[DW] R. Dijkgraaf and E. Witten, Topological gauge theories and group cohomology, Commun. Math. Phys. 129 (1990) 393-429

[ENO] P.I. Etingof, D. Nikshych, and V. Ostrik, An analogue of Radford's $S^{4}$ formula for finite tensor categories, Int. Math. Res. Notices (2004) 2915-2933 [math.QA/0404504]

[Fr] D.S. Freed, Classical Chern-Simons theory, Part 1, Adv. Math. 113 (1995) 237-303 [hep-th/9206021]

[FFRS] J. Fröhlich, J. Fuchs, I. Runkel, and C. Schweigert, Duality and defects in rational conformal field theory, Nucl. Phys. B 763 (2007) 354-430 [hep-th/0607247]

[FNSW] J. Fuchs, T. Nikolaus, C. Schweigert, and K. Waldorf, Bundle gerbes and surface holonomy, in: European Congress of Mathematics, A.C.M. Ran, H. te Riele, and J. Wiegerinck, eds. (European Math. Society, Zürich 2010), p. 167-195 [math.DG/0901.2085]

[FSV] J. Fuchs, C. Schweigert, and A. Valentino, Bicategories for boundary conditions and for surface defects in 3-d TFT, Commun. Math. Phys. 321 (2013) 543-575 [hep-th/1203.4568]

[FSW] J. Fuchs, C. Schweigert, and K. Waldorf, Bi-branes: Target space geometry for world sheet topological defects, J. Geom. and Phys. 58 (2008) 576-598 [hep-th/0703145]

[Ka] A. Kapustin, Ground-state degeneracy for abelian anyons in the presence of gapped boundaries, Phys. Rev. B 89 (2014) 125307-1-7 [cond-mat/1306.4254]

[KaS] A. Kapustin and N. Saulina, Surface operators in 3d topological field theory and 2d rational conformal field theory, in: Mathematical Foundations of Quantum Field and Perturbative String Theory, H. Sati and U. Schreiber, eds. (American Mathematical Society, Providence 2011), p. 175-198 [hep-th/1012.0911]

[KK] A. Kitaev and L. Kong, Models for gapped boundaries and domain walls, Commun. Math. Phys. 313 (2012) 351-373 [cond-mat/1104.5047]

[LaP] A.D. Lauda and H. Pfeiffer, Open-closed strings: Two-dimensional extended TQFTs and Frobenius algebras, Topology Applic. 155 (2008) 623-666 [math.AT/0510664]

[Le] M. Levin, Protected edge modes without symmetry, Phys. Rev. X 3 (2013) 021009-1-18 [cond-mat/1301.7355]

[MoS] G. Moore and G. Segal, D-branes and K-theory in 2D topological field theory, in: Dirichlet Branes and Mirror Symmetry, P. Aspinwall et al. (American Mathematical Society, Providence 2009), p. 27-108 [hep-th/0609042]

[Mor] J.C. Morton, Extended TQFT, gauge theory, and 2-linearization, preprint math.QA/ 1003.5603

[Os1] V. Ostrik, Module categories, weak Hopf algebras and modular invariants, Transform. Groups 8 (2003) 177-206 [math.QA/0111139]

[Os2] V. Ostrik, Module categories over the Drinfeld double of a finite group, Int. Math. Res. Notices No. 27 (2003) 1507-1520 [math.QA/0202130]

[SFR] C. Schweigert, J. Fuchs, and I. Runkel, Categorification and correlation functions in conformal field theory, in: Proceedings of the ICM 2006, M. Sanz-Solé, J. Soria, J.L. Varona, and J. Verdera, eds. (European Math. Society, Zürich 2006), p. 443-458 [math.CT/0602079] 
[St] N. Steenrod, The Topology of Fiber Bundles (Princeton University Press, Princeton 1951)

[WW] J. Wang and X.-G. Wen, Boundary degeneracy of topological order, preprint cond-mat/ 1212.4863

[Wi] S. Willerton, The twisted Drinfeld double of a finite group via gerbes and finite groupoids, Alg. \& Geom. Topol. 8 (2008) 1419-1457 [math.QA/0503266] 